\title{
24. FINE-FRACTION CARBONATE OXYGEN AND CARBON ISOTOPE RESULTS FROM SITE 704: IMPLICATIONS FOR MOVEMENT OF THE POLAR FRONT DURING THE LATE PLIOCENE ${ }^{1}$
}

\author{
Gregory A. Mead, ${ }^{2}$ David A. Hodell, ${ }^{2}$ Daniel W. Müller, ${ }^{3}$ and Paul F. Ciesielski ${ }^{2}$
}

\begin{abstract}
Ocean Drilling Program Site 704 in the subantarctic South Atlantic was drilled to investigate the response of the Southern Ocean to climatic and oceanographic developments during the late Neogene.

Stable oxygen and carbon isotopes of fine-fraction $(<63 \mu \mathrm{m})$ carbonate were analyzed to supplement similar analyses of benthic and planktonic foraminifers. The fine fraction is generally composed primarily of coccoliths, and isotopic analyses of the fine fraction were made to complement the foraminiferal analyses. The isotopic curves thus generated suggest paleoceanographic changes not recognizable by the use of benthic and planktonic foraminifers alone.

The global Chron 6 carbon isotope shift, found at 253-244 mbsf (6.39-6.0 Ma) at Site 704 in the planktonic and benthic record, is seen in the fine-fraction $\delta^{13} \mathrm{C}$ record as a gradual decrease from 255 mbsf (6.44 Ma) to 210 mbsf (4.24 Ma).

At 170 mbsf, mean $\delta^{18} \mathrm{O}$ values of Neogloboquadrina pachyderma increase by $0.6 \% 0-0.7 \%$ (Hodell and Ciesielski, this volume), reflecting decreased temperature and increased continental ice volume. Accumulation rates increase by 3.3 times above this depth (which corresponds to an age of $2.5 \mathrm{Ma}$ ), suggesting increased upwelling and biologic productivity.

Carbon isotopic values of fine-fraction carbonate decrease by about $1.5 \%$ at $2.6 \mathrm{Ma}$; however, no change is recorded in the $\delta^{13} \mathrm{C}$ of $N$. pachyderma. The fine-fraction $\delta^{13} \mathrm{C}$ shift slightly precedes an average $1 \%$ decrease in $\delta^{13} \mathrm{C}$ in benthic foraminifers. The cause of the benthic $\delta^{13} \mathrm{C}$ shift (most likely due to a change in deep water circulation; Hodell and Ciesielski, this volume) is probably not directly related to the fine-fraction shift. The fine-fraction shift is most likely caused by (1) a change in the upwelling to productivity ratio at this site, with increased upwelling bringing lighter carbon to surface waters, more productivity, and higher sedimentation rates and (2) a change in the particle composition of the fine fraction. The increased upwelling is probably due to a northward migration of the Antarctic Polar Front to a position nearer Site 704.
\end{abstract}

\section{INTRODUCTION}

Ocean Drilling Program (ODP) Site 704 was drilled on the Meteor Rise at $46^{\circ} 53^{\prime} \mathrm{S}, 7^{\circ} 25^{\prime} \mathrm{E}$, at a depth of $2532 \mathrm{~m}$ (Fig. 1). The site is just north of the present position of the Antarctic Polar Front Zone (APFZ), and south of the Subtropical Convergence, as defined by the expendable bathythermograph data of Lutjeharms (1985). Site 704 recovered a nearly continuous section from the middle Miocene through the latest Pleistocene using hydraulic piston corer and extended core barrel coring techniques in two adjacent holes, with carbonate content ranging from $0 \%$ to $96 \%$. Paleomagnetic and biostratigraphic analyses show that the only gap in the Pliocene-Pleistocene section is a possible compressed inter$\mathrm{val}$ in the Brunhes. The upper $50 \mathrm{~m}$ of section is slightly to severely disturbed, however, so the age of this interval is not well constrained. Carbon and oxygen isotope analyses of planktonic and benthic foraminifers (see Hodell et al., this volume; Müller et al., this volume) and the bulk fine fraction $(>63 \mu \mathrm{m})$ have been used to examine paleoceanographic conditions in the South Atlantic during the late Neogene.

The history of climatic change through the Cenozoic has been characterized by periods of relative stasis punctuated by sudden episodes of change (Shackleton and Kennett, 1975). These events are recorded in oxygen and carbon isotopic

\footnotetext{
${ }^{1}$ Ciesielski, P. F., Kristoffersen, Y., et al., 1991. Proc. ODP, Sci. Results, 114: College Station, TX (Ocean Drilling Program).

2 Department of Geology, University of Florida, Gainesville, FL 32611.

${ }^{3}$ Geologisches Institut, ETH-Zentrum, CH-8092 Zürich, Switzerland.
}

records from the deep sea and can help us understand the causes and effects of these changes.

The Neogene was the time during which major, permanent ice caps developed and during which the present glacialinterglacial cycles were initiated in the Northern Hemisphere. Although small valley glaciers or temporary ice caps may have occurred on Antarctica during the Oligocene (Shackleton and Kennett, 1975; Matthews and Poore, 1980; Miller and Fairbanks, 1983; Miller et al., 1985), a permanent ice cap on East Antarctica did not develop until the mid-Miocene, at about 14 $\mathrm{Ma}$ (Shackleton and Kennett, 1975). Deep Sea Drilling Project (DSDP) drilling at several sites in the North Atlantic has provided evidence that significant Northern Hemisphere glaciation began in the late Pliocene (Shackleton et al., 1984; Raymo et al., 1989). Shackleton et al. (1984) first presented evidence that ice-rafted debris began to accumulate in the North Atlantic at DSDP Site 552 at about $2.4 \mathrm{Ma}$, while carbonate concentration in the sediment decreased due to the diluent effect of ice-rafted debris. In addition, isotopic analyses of benthic foraminifers revealed an average ${ }^{18} \mathrm{O}$ enrichment in biogenic calcite of over $1.0 \%$, indicating the accumulation of isotopically light continental ice. The initial glacial episodes resulted in oxygen isotope fluctuations of about $1 \%$, at periods of approximately $41,000 \mathrm{yr}$, corresponding to Milankovitch obliquity variation (Ruddiman et al., 1986). Raymo et al. (1989) found small amounts of ice-rafted debris present in sediments as old as $2.65 \mathrm{Ma}$ in DSDP Hole 607 . Spectral analysis of oxygen isotopic variations of Pliocene sediments from Hole 607 also indicates periodicities of about $41,000 \mathrm{yr}$, although with a lower amplitude than after $2.4 \mathrm{Ma}$. By the Brunes/Matuyama boundary, the amplitude of the $\delta^{18} \mathrm{O}$ variations had increased to about $2 \%$, and the dominant 


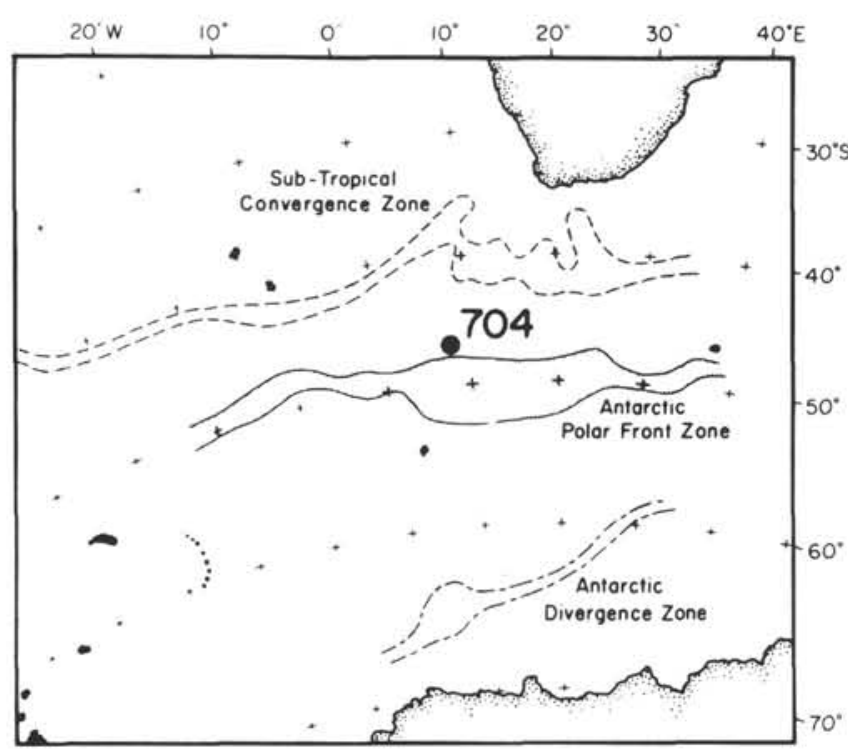

Figure 1. The position of ODP Site 704 in the eastern subantarctic South Atlantic relative to the major hydrographic fronts (modified after Lutjeharms, 1985).

period of the glacial-interglacial cycles increased to approximately 100,000 yr (Shackleton et al., 1984; Ruddiman et al., 1986, 1989).

The onset of large-scale Northern Hemisphere glaciation is a key event in the development of the Earth's present climatic regime, with cyclic glaciations characterizing the Earth's climate during the Pleistocene and late Pliocene. However, the interaction of the Southern Hemisphere with the Northern Hemisphere at the onset of these glaciations is not well understood.

\section{Stable Isotopic Studies of Fine-Fraction Carbonate}

Several studies have examined the utility of calcareous nannofossil isotopic data as paleoceanographic indicators (Anderson and Cole, 1975; Margolis et al., 1975; Goodney et al., 1980; Dudley et al., 1980, 1986; Paull and Theirstein, 1987). Results of these studies have been inconclusive because several variables control the isotopic composition of the fine fraction. Margolis et al. (1975) found that nannofossil $\delta^{18} \mathrm{O}$ was roughly equivalent to that of planktonic foraminifers, whereas $\delta^{13} \mathrm{C}$ of nannofossil calcite exhibited a variable relationship to planktonic foraminiferal $\delta^{13} \mathrm{C}$. Goodney et al. (1980) investigated the isotopic response of calcareous nannofossils in Holocene sediments. They found that $\delta^{18} \mathrm{O}$ and $\delta^{13} \mathrm{C}$ of the fine fraction covaried, with a slope near 1. They suggested that the trend was caused by assemblage changes in the fine fraction. Paull and Theirstein (1987) found that different grain-size subfractions of the fine fraction from the same sample (roughly corresponding to different taxonomic groups) had different $\delta^{18} \mathrm{O}$ and $\delta^{13} \mathrm{C}$ values. The isotopic compositions of the subfractions lay along a linear trend of increasing $\delta^{18} \mathrm{O}$ with increasing $\delta^{13} \mathrm{C}$, similar to that of Goodney et al. (1980).

Another finding of Goodney et al. (1980) was the existence of a latitudinal pattern to the carbon isotopic gradient between planktonic foraminifers and nannofossils. They found that when $\delta^{13} \mathrm{C}$ of planktonic foraminifers increased, $\delta^{13} \mathrm{C}$ of calcareous nannofossils decreased, increasing the gradient between them, and, in addition, that this effect varied systematically with latitude. They suggested that the mirrored relationship was due to effects of changing rates of productivity on $\delta^{13} \mathrm{C}$ of $\Sigma \mathrm{CO}_{2}$. In low-productivity areas, photosynthesis would have little effect on $\delta^{13} \mathrm{C}$, and the $\delta^{13} \mathrm{C}$ values of planktonic foraminifers and the fine fraction would be similar. In areas of high productivity, photosynthesis preferentially extracts ${ }^{12} \mathrm{C}$, increasing $\delta^{13} \mathrm{C}$ and causing the $\delta^{13} \mathrm{C}$ of planktonic foraminifers to be elevated relative to that of the fine fraction. Dudley et al. (1980) suggested that because coccolith calcite deposition is intracellular, metabolic $\mathrm{CO}_{2}$ may be incorporated into the calcite, and Goodney et al. (1980) suggested that nannofossil $\delta^{13} \mathrm{C}$ would be lower for that reason.

In the Southern Ocean, conditions controlling $\delta^{13} \mathrm{C}$ of surface waters are somewhat different. Insolation in the Antarctic area is less than in more temperate areas because of the low angle of the sun. Furthermore, temperatures are low, slowing biologic processes. Primary productivity in this area is thus light-limited rather than nutrient-limited (Knox and McElroy, 1984). Therefore, surface waters in areas of high productivity in high latitudes tend to be characterized by low $\delta^{13} \mathrm{C}$ values because the balance between preferential removal of ${ }^{12} \mathrm{C}$ by photosynthesis and carbon with low $\delta^{13} \mathrm{C}$ values supplied by upwelling/mixing will be in shifted toward the supply side. In areas of high productivity caused by increased upwelling, the effect of photosynthesis on $\delta^{13} \mathrm{C}$ would be swamped by the upwelling effect. In areas of low productivity caused by slower replenishment of carbon and other nutrients, nearly all carbon would be extracted by photosynthesis, producing greatly enriched $\delta^{13} \mathrm{C}$. Carbon isotopic compositions of surface waters thus depend on the balance between the rate of carbon removed because of photosynthesis and the rate of ${ }^{12} \mathrm{C}$-enriched carbon supplied by upwelling and surface currents.

Because of this balance and other factors discussed subsequently, $\delta^{13} \mathrm{C}$ gradients exist in the surface waters of the Antarctic, with lower $\delta^{13} \mathrm{C}$ values occurring in the high-productivity area south of the Polar Front (Kroopnick, 1980, 1985). Carbon isotopic composition of surface water is also affected by exchange with the atmosphere. Charles and Fairbanks (1990) have shown that there is a decrease in $\delta^{13} \mathrm{C}$ both north and south of the Polar Front. They suggest that these gradients result not only from upwelling/productivity effects but also from the rate of gas exchange and the temperature-dependent nature of the gas exchange fractionation north of the front and from gas exchange fractionation and incomplete equilibration of surface waters with the atmosphere south of the front.

This paper will discuss results of oxygen and carbon isotopic analyses of fine-fraction carbonate samples from ODP Site 704 in the South Atlantic, which suggest that

1. a northward migration of the Antarctic Polar Front over this site occurred shortly before the onset of Northern Hemisphere glaciation, leading to

2 . increasing productivity, and

3. a change in the $\delta^{13} \mathrm{C}$ of pelagic carbonate at this site.

\section{METHODS}

\section{Age-Depth Model}

The time scale used in this report is calibrated to the Berggren et al. (1985) time scale. The $S$ terminology for paleomagnetic boundaries is from Hailwood (1989). The tie points shown in Table 1 are from Hailwood and Clement (this volume), and other dates were obtained by linear interpolation between these points.

\section{Sampling Strategy}

Holes 704A and 704B were both used to construct the isotope section. Samples were used from the upper $25 \mathrm{~m}$ of both holes. Core 114-704A-4H was highly disturbed, so sam- 
Table 1. Age-depth model from Hailwood and Clement (this volume). The $S$ terminology is from Hailwood (1989); dates are from Berggren et al. (1985).

\begin{tabular}{lll}
\hline $\begin{array}{l}\text { Depth } \\
\text { (mbsf) }\end{array}$ & $\begin{array}{c}\text { Age } \\
\text { (Ma) }\end{array}$ & \\
\hline 0.00 & 0.00 & Seafloor \\
1.14 & 0.195 & LAAD Hemidiscus karstenii \\
15.55 & 0.62 & LAD Actinocyclus ingens \\
34.51 & 0.73 & Brunhes/Matuyama (S1N/S1R) boundary \\
44.50 & 0.98 & Base Jaramillo \\
87.40 & 1.66 & Top Olduvai \\
100.25 & 1.89 & Base Olduvai \\
168.72 & 2.47 & Matuyama/Gauss (S2R/S3N) boundary \\
169.13 & 2.64 & LAD Nitzschia weaveri \\
176.70 & 2.92 & Top Kaena \\
177.98 & 2.99 & Base Kaena \\
178.96 & 3.08 & Top Mammoth \\
181.13 & 3.18 & Base Mammoth \\
186.69 & 3.40 & Gauss/Gilbert (S3N/S3R) boundary \\
195.79 & 3.88 & Top Cochiti (S3R/S4N boundary) \\
201.94 & 3.97 & Base Cochiti (S4N.10) \\
204.19 & 4.10 & Top Nunivak (S4N.25) \\
210.29 & 4.24 & Base Nunivak (S4N.40) \\
211.99 & 4.40 & Top C1 (S4N.58) \\
213.22 & 4.47 & Base C1 (S4N.66) \\
217.29 & 4.57 & S4N.78 \\
219.44 & 4.77 & S4N/S4R boundary \\
224.75 & 5.15 & FAD Thalassiosia oestrupii \\
224.89 & 5.35 & S4R/S5N boundary \\
231.00 & 5.53 & S5N.33 \\
234.00 & 5.68 & S5N.61 \\
241.50 & 5.89 & S5N/S5R boundary \\
252.20 & 6.37 & SSR.59 \\
257.50 & 6.50 & S5R.75 \\
259.50 & 6.70 & SSR/S6N boundary \\
264.50 & 6.78 & S6N.11 \\
265.50 & 6.85 & S6N.21 \\
267.50 & 7.28 & S6N.82 \\
270.20 & 7.35 & S6N.92 \\
275.20 & 7.41 & S6N/S6R boundary \\
290.00 & 7.90 & S6R/S7N boundary \\
308.50 & 8.21 & S7N.52 \\
309.50 & 8.41 & S7N.85 \\
318.50 & 8.50 & S7N/S7R boundary \\
324.00 & 8.71 & S7R.50 \\
327.00 & 8.80 & S7R.71 \\
338.50 & 8.92 & S7R/S8N boundary \\
379.95 & 10.40 & S8N/S8R boundary \\
\hline & &
\end{tabular}

${ }^{a}$ LAAD $=$ last absolute appearance datum $;$ LAD $=$ last appearance datum; FAD = first-appearance datum.

ples from Hole 704B only were used from 25.41 to $34.26 \mathrm{~m}$ below seafioor (mbsf). Color variation (Shipboard Scientific Party, 1988) and carbonate content (Froelich et al., this volume) in the upper part of the section show little correlation between the holes. Because of this, no attempt was made to splice samples from the two holes. Instead, the data from each hole are plotted on the figures separately, but on a common depth scale. Sample recovery was low or zero in cores from Hole 704A below Core 114-704A-25X (235.2 mbsf), so samples from Holes 704A and 704B were overlapped from 213.91 to 233.41 mbsf. Carbonate data (Froelich et al., this volume) show a variable offset in depth between the holes of up to $1 \mathrm{~m}$, but no consistent correlation is possible based on these data. Therefore, again, the data are plotted separately on a common depth scale. Samples from Hole 704B were used exclusively from 233.81 mbsf to the deepest fine-fraction sample at 289.66 mbsf, with an age of $7.89 \mathrm{Ma}$.

The sampling intervals vary with depth. Within the upper $80 \mathrm{~m}$, the sampling interval averages $30.9 \mathrm{~cm}$, or one per 6000 yr. Between 80 and $168 \mathrm{mbsf}$, the average is $145.8 \mathrm{~cm}$, or one per $15,000 \mathrm{yr}$. The average sampling interval from 168 to 200 mbsf is about the same, $166.7 \mathrm{~cm}$, but because of a sedimen- tation rate change, the average sample frequency is only one per $77,500 \mathrm{yr}$. From 200 to $290 \mathrm{mbsf}$, the sampling interval is smaller, averaging $37.8 \mathrm{~cm}$, and the sampling frequency averages one per $16,800 \mathrm{yr}$.

\section{Isotopic Analyses}

The fine-fraction $(<63 \mu \mathrm{m})$ samples of Site 704 were obtained by disaggregating bulk samples in a water solution of warm sodium hexa-metaphosphate and washing through a $63-\mu \mathrm{m}$ sieve. The coarse fraction was saved for isotopic analyses of foraminifers (Hodell et al., this volume). The fine-fraction residues were collected and settled for at least 24 hr. Excess water was poured off, and the fine-fraction was concentrated by centrifugation and dried in a $50^{\circ} \mathrm{C}$ oven. Fine-fraction samples were mechanically homogenized, and a small subsample was roasted at $400^{\circ} \mathrm{C}$ in a vacuum for $1 \mathrm{hr}$ to remove organic carbon. The subsamples were reacted in a common $100 \%$ orthophosphoric acid bath at $70^{\circ} \mathrm{C}$ to release $\mathrm{CO}_{2}$, which was analyzed in an automated triple collector VG Isogas Prism mass spectrometer. Isotopic results were corrected to $\mathrm{PDB}$ via a set of intermediate standards (Hodell et al., 1989). Precision of replicate fine-fraction analyses averaged $0.16 \%$ for oxygen and $0.13 \%$ for carbon. Isotopic results that differed greatly from the analysis of neighboring samples were reanalyzed for confirmation. If repeat analyses differed by more than $0.5 \%$, a third replicate was analyzed; analyses that differed from the other two by more than $0.5 \%$ were rejected, and if all three were within $0.5 \%$, all were accepted.

Barrera and Savin (1987) investigated the effect of isotopic exchange between the fine fraction and evaporating wash water. They found that substantial effects, caused by Rayleigh fractionation, occurred in the very fine fraction $(<10 \mu \mathrm{m})$ that they analyzed. To investigate whether any similar effects occurred with the Site 704 samples, six fine-fraction samples were resuspended after initial centrifugation, split, and the water replaced with methanol in one of the splits through the use of three successive resuspensions and centrifuge concentrations. The results are shown in Table 2. The oxygen isotopes of the water-dried fraction differ from those of the methanol-dried fraction by an average of $0.01 \% \pm 0.07 \%$ (two standard deviations), and carbon isotopes differ by an average of $0.02 \pm 0.10 \%$ (two standard deviations). We conclude that the samples used in this study were not affected by Rayleigh fractionation during drying.

Analytic precision as established by repeat analyses of an internal working standard (Carrara Marble) is $0.13 \%$ for oxygen and $0.04 \%$ for carbon isotopes.

\section{Fine-Fraction Compositional Analysis}

One objective of this study was to determine whether compositional changes in the fine-fraction assemblage caused changes in the isotopic composition. Portions of the homogenized fine fraction were selected from samples at approximately $10-\mathrm{m}$ intervals. Slides of these samples were used to estimate fine-fraction composition. The method of Moore (1973) was used to make slides with randomly settled grains, to ensure a representative estimate of fine-fraction composition. The percentage of three calcareous particle types (foraminifers, coccoliths, and micrite) was estimated from each slide, with the least common particle type estimated first, and the most common type calculated by the difference between $100 \%$ and the sum of the other two particle types.

\section{RESULTS}

The fine-fraction carbonate isotope record shows significant variability in both carbon and oxygen isotopes (Fig. 2 and 
Table 2. Effects of methanol drying vs. water drying on the isotopic content of the fine fraction.

\begin{tabular}{|c|c|c|c|c|c|c|}
\hline \multirow[b]{2}{*}{$\begin{array}{l}\text { Core, section, } \\
\text { interval }(\mathrm{cm})\end{array}$} & \multicolumn{3}{|c|}{$\delta^{18} \mathrm{O}$} & \multicolumn{3}{|c|}{$\delta^{13} \mathrm{C}$} \\
\hline & $\begin{array}{l}\text { Water } \\
\text { dried }\end{array}$ & $\begin{array}{c}\text { Methanol } \\
\text { dried }\end{array}$ & $\begin{array}{l}\text { Difference } \\
\text { between } \\
\text { methods }\end{array}$ & $\begin{array}{l}\text { Water } \\
\text { dried }\end{array}$ & $\begin{array}{c}\text { Methanol } \\
\text { dried }\end{array}$ & $\begin{array}{l}\text { Difference } \\
\text { between } \\
\text { methods }\end{array}$ \\
\hline 114-704A- & & & & & & \\
\hline $19 X-2,21$ & 2.92 & 2.89 & 0.03 & 0.92 & 0.88 & 0.04 \\
\hline $19 X-4,21$ & 2.54 & 2.57 & -0.03 & 0.45 & 0.51 & -0.06 \\
\hline $20 X-2,21$ & 2.58 & 2.55 & 0.03 & 0.54 & 0.59 & -0.05 \\
\hline $20 X-6,21$ & 2.16 & 2.10 & 0.06 & 0.51 & 0.60 & -0.09 \\
\hline $21 X-2,46$ & 2.06 & 2.09 & -0.03 & 0.44 & 0.44 & 0.00 \\
\hline $22 X-2,21$ & 2.24 & 2.23 & 0.01 & 0.76 & 0.73 & 0.03 \\
\hline Mean & & & 0.01 & & & -0.02 \\
\hline
\end{tabular}

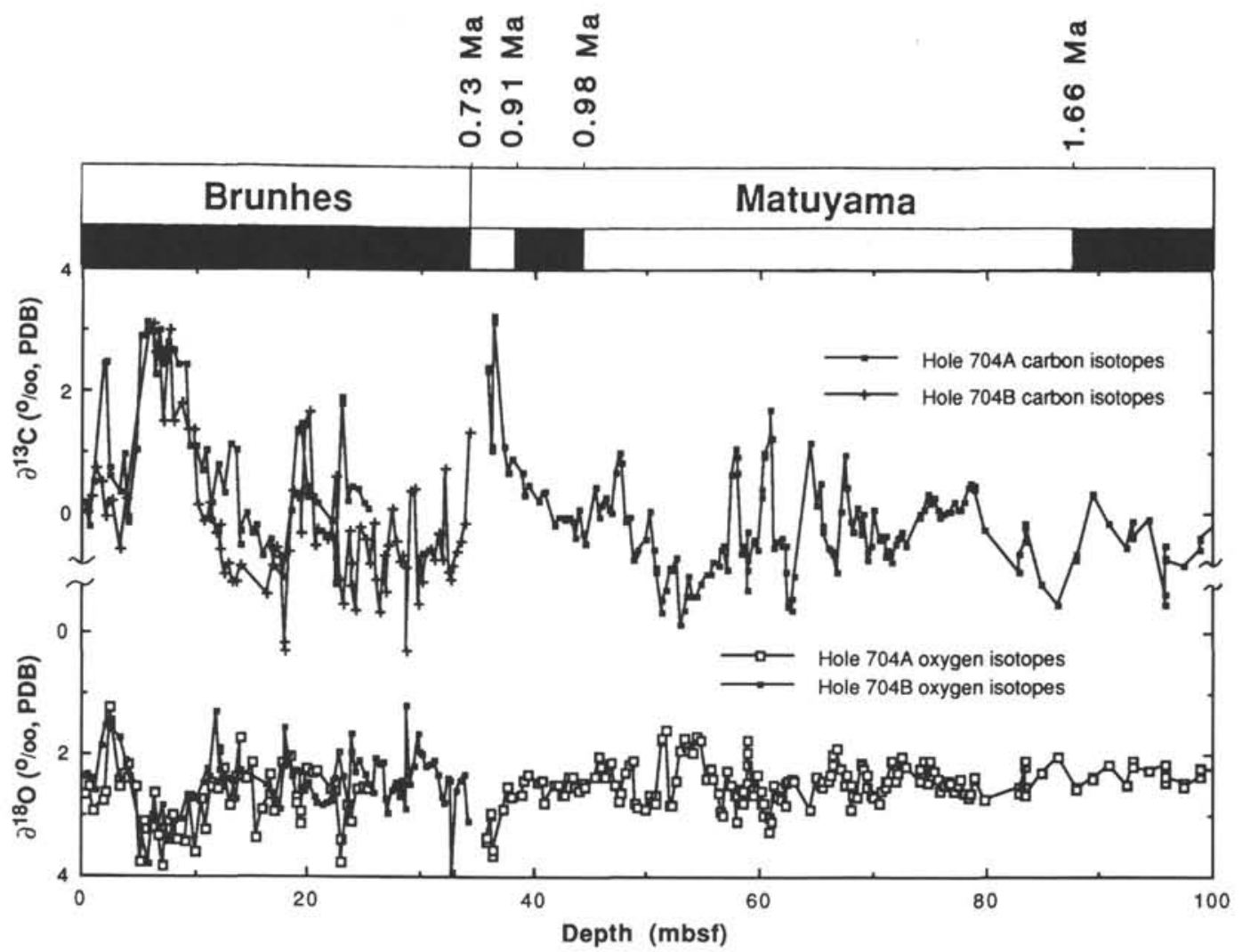

Figure 2. Fine-fraction $\delta^{13} \mathrm{C}$ and $\delta^{18} \mathrm{O}$ records vs. depth, $0-300 \mathrm{mbsf}$.

Table 3). The maximum amplitude in both isotope signals occurs in the upper $40 \mathrm{~m}$, with a range of $5.53 \%(-2.3 \%$ to $3.23 \%$ ) for $\delta^{13} \mathrm{C}$ and $2.77 \%$ oo $\left(1.18 \%\right.$ oo to $3.95 \%$ oo ) for $\delta^{18} \mathrm{O}$.

Covariance between $\delta^{13} \mathrm{C}$ and $\delta^{18} \mathrm{O}$ occurs over much of the fine-fraction record (Fig. 3). These results contrast with the trend line found by Goodney et al. (1980) of much stronger covariance between $\delta^{13} \mathrm{C}$ and $\delta^{18} \mathrm{O}$ in the tops of cores from the Indian Ocean. However, it is important to remember that the core-top data of Goodney et al. (1980) were not affected by ice volume changes, whereas downcore data will be. Simultaneous maxima in $\delta^{13} \mathrm{C}$ and $\delta^{18} \mathrm{O}$ occur at several levels: 238.9-240.4 mbsf (5.8 Ma), 221.2 mbsf (4.90 Ma), 203.5 mbsf (4.06 Ma), $187.4 \mathrm{mbsf}(3.44 \mathrm{Ma}), 181.4 \mathrm{mbsf}(3.19 \mathrm{Ma})$, and 175 mbsf (2.86 Ma).

Note that the data from Holes 704A and 704B correspond very well in the areas of overlap (0-36.2 and 213.9-233.4 mbsf) even without splicing. This suggests that little displace- ment of sediment has occurred in the disturbed upper section. The isotopic signal is small in the lower region of overlap, and thus little difference in values is seen there despite the variable $1-\mathrm{m}$ offset previously mentioned.

\section{Oxygen Isotopes}

The $\delta^{18} \mathrm{O}$ record exhibits less variability than the $\delta^{13} \mathrm{C}$ record (Fig. 2). From about $290 \mathrm{mbsf}$ (7.9 Ma) to $264-253 \mathrm{mbsf}$ (6.77-6.39 Ma), the oxygen isotopic composition of the fine fraction became gradually more enriched in ${ }^{18} \mathrm{O}$, from an average of $2.44 \%$ between 290 and $280 \mathrm{mbsf}$ to an average of $2.82 \%$ between 264 and 253 mbsf. At this point, $\delta^{18} \mathrm{O}$ began to decrease over the next $40 \mathrm{~m}(1.7 \mathrm{~m}$.y.), to about $2 \%$ at 222 mbsf $(4.95 \mathrm{Ma})$. There is one short period of enrichment in ${ }^{18} \mathrm{O}$ at about $240 \mathrm{mbsf}(5.85 \mathrm{Ma})$, with $\delta^{18} \mathrm{O}$ increasing to more than $3 \%$. From 222 to about 180 mbsf (3.13 Ma), a slow, uneven $\delta^{18} \mathrm{O}$ enrichment occurs, rising from about $2 \%$ to about 


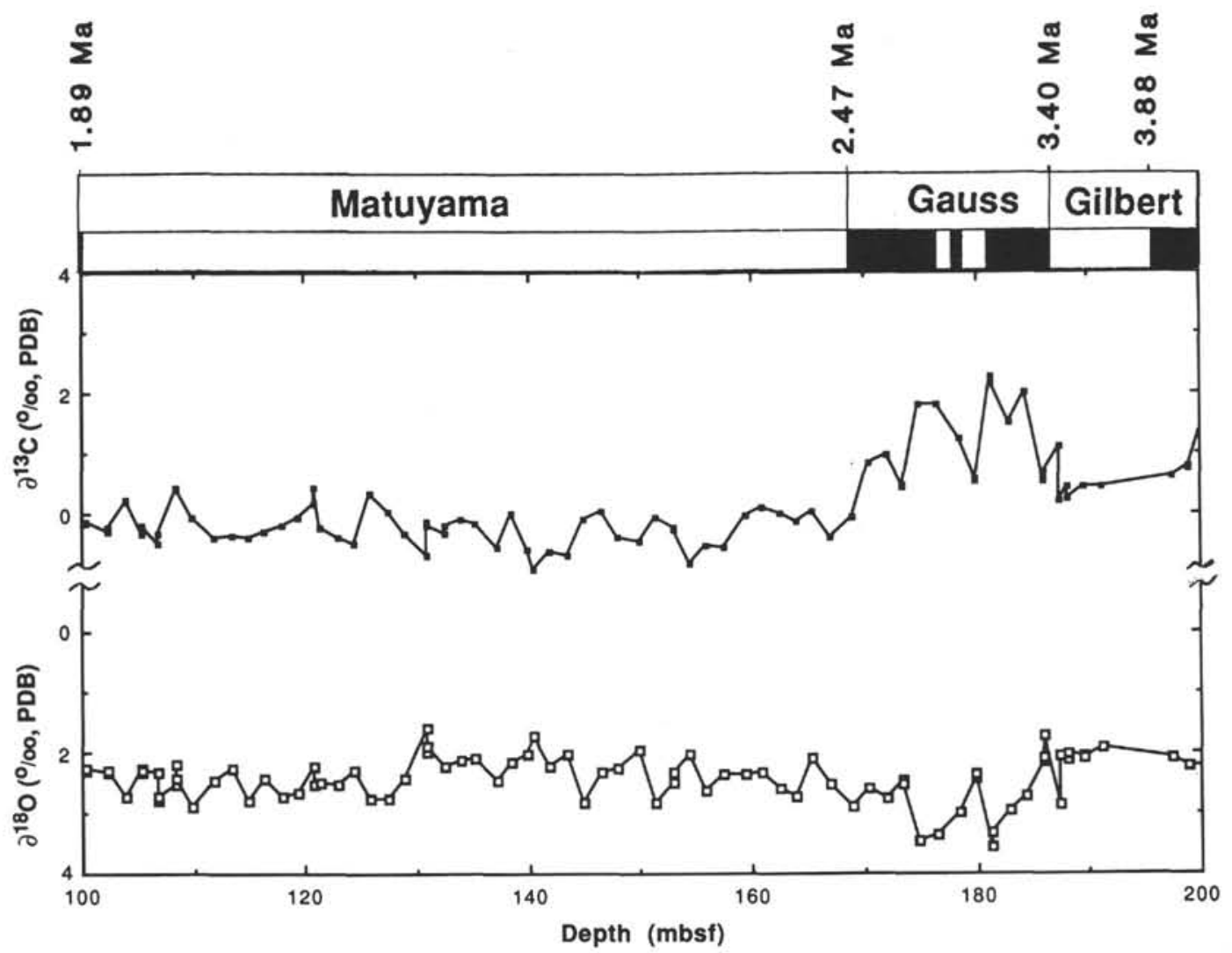

Figure 2 (continued).

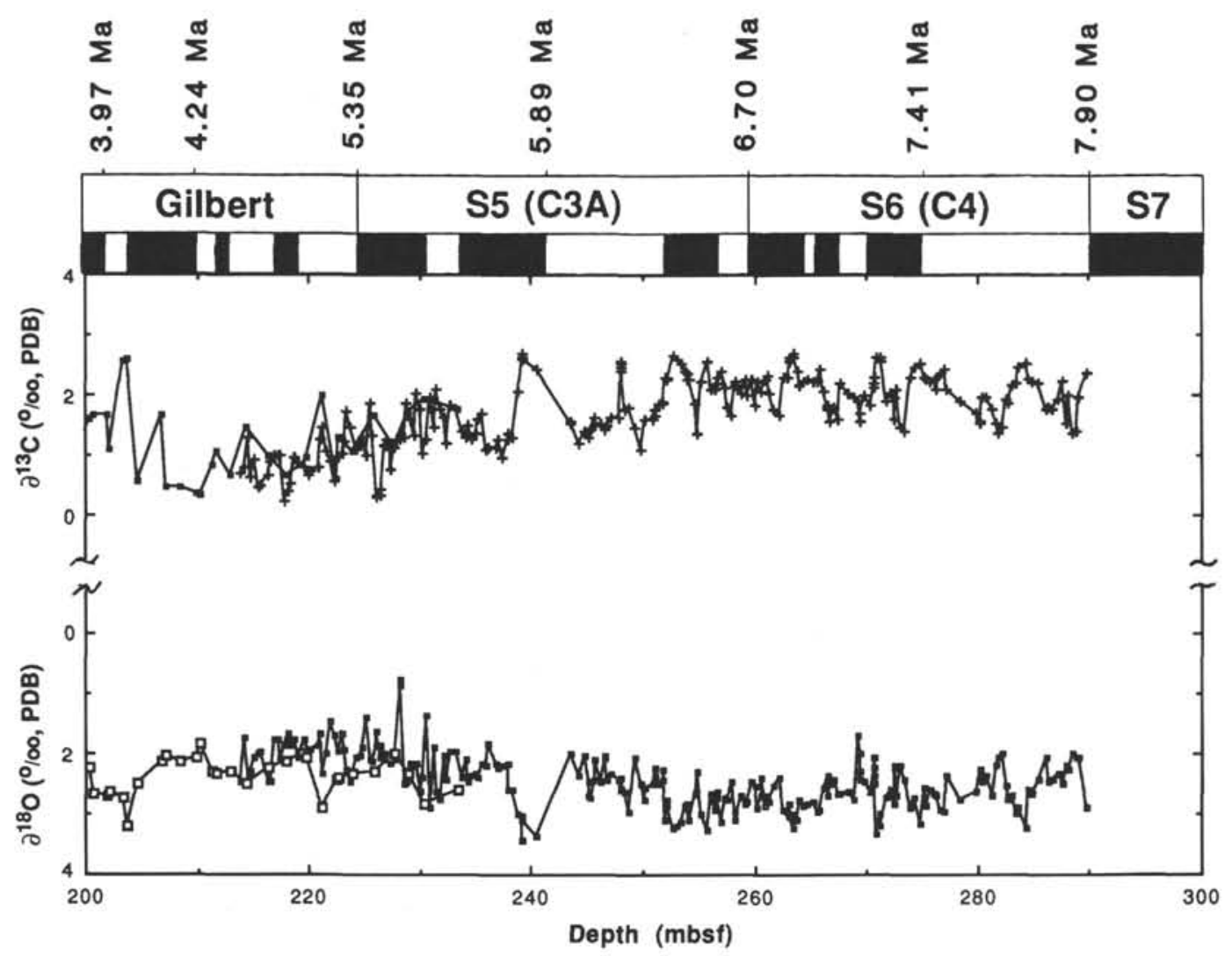

Figure 2 (continued). 
Table 3. Fine-fraction $\delta^{13} \mathrm{C}$ and $\delta^{18} \mathrm{O}$ values, Site 704.

\begin{tabular}{|c|c|c|c|c|}
\hline $\begin{array}{l}\text { Core, section, } \\
\text { interval }(\mathrm{cm})\end{array}$ & $\begin{array}{l}\text { Depth } \\
\text { (mbsf) }\end{array}$ & $\begin{array}{l}\text { Age } \\
\text { (Ma) }\end{array}$ & $\delta^{13} \mathrm{C}$ & $\delta^{18} \mathrm{O}$ \\
\hline 114-704A- & & & & \\
\hline $1 \mathrm{H}-1,41$ & 0.41 & 0.0701 & 0.158 & 2.678 \\
\hline $1 \mathrm{H}-1,78$ & 0.78 & 0.1334 & -0.236 & 2.516 \\
\hline $1 \mathrm{H}-1,110$ & 1.10 & 0.1882 & 0.486 & 2.939 \\
\hline $1 \mathrm{H}-2,41$ & 1.91 & 0.2177 & 2.432 & 2.750 \\
\hline $1 \mathrm{H}-2,78$ & 2.22 & 0.2269 & 2.473 & 2.637 \\
\hline $1 \mathrm{H}-2,110$ & 2.60 & 0.2381 & 0.573 & 1.223 \\
\hline $1 \mathrm{H}-2,110$ & 2.60 & 0.2381 & 0.720 & 1.461 \\
\hline $1 \mathrm{H}-3,41$ & 3.41 & 0.2620 & 0.333 & 2.545 \\
\hline $1 \mathrm{H}-3,41$ & 3.41 & 0.2620 & 0.337 & 2.427 \\
\hline $1 \mathrm{H}-3,78$ & 3.78 & 0.2729 & 0.950 & 2.321 \\
\hline $1 \mathrm{H}-3,78$ & 3.78 & 0.2729 & 0.950 & 2.321 \\
\hline $1 \mathrm{H}-3,110$ & 4.10 & 0.2823 & -0.087 & 2.166 \\
\hline $1 \mathrm{H}-3,110$ & 4.10 & 0.2823 & -0.192 & 2.382 \\
\hline $1 \mathrm{H}-4,41$ & 4.91 & 0.3062 & 1.015 & 2.536 \\
\hline $1 \mathrm{H}-4,78$ & 5.28 & 0.3171 & 2.907 & 3.769 \\
\hline $1 \mathrm{H}-4,110$ & 5.60 & 0.3265 & 2.897 & 3.108 \\
\hline $1 \mathrm{H}-4,125$ & 5.75 & 0.3310 & 3.138 & 3.224 \\
\hline $1 \mathrm{H}-5,21$ & 6.21 & 0.3445 & 2.970 & 3.201 \\
\hline $1 \mathrm{H}-5,41$ & 6.41 & 0.3504 & 2.263 & 2.620 \\
\hline $1 \mathrm{H}-5,78$ & 6.78 & 0.3613 & 3.010 & 3.324 \\
\hline $1 \mathrm{H}-5,110$ & 7.10 & 0.3708 & 2.434 & 3.838 \\
\hline $2 \mathrm{H}-1,41$ & 7.61 & 0.3858 & 2.801 & 3.097 \\
\hline $2 \mathrm{H}-1,82$ & 8.02 & 0.3979 & 2.652 & 2.990 \\
\hline $2 \mathrm{H}-1,132$ & 8.52 & 0.4127 & 2.423 & 3.404 \\
\hline $2 \mathrm{H}-2,41$ & 9.11 & 0.4301 & 2.437 & 3.436 \\
\hline $2 \mathrm{H}-2,82$ & 9.52 & 0.4422 & 1.106 & 2.682 \\
\hline $2 \mathrm{H}-2,132$ & 10.02 & 0.4569 & 1.075 & 3.588 \\
\hline $2 \mathrm{H}-3,4 \mathrm{I}$ & 10.61 & 0.4743 & 0.677 & 2.742 \\
\hline $2 \mathrm{H}-3,82$ & 11.02 & 0.4864 & 1.025 & 3.221 \\
\hline $2 \mathrm{H}-3,132$ & 11.52 & 0.5011 & 0.166 & 2.528 \\
\hline $2 \mathrm{H}-3,132$ & 11.52 & 0.5011 & -0.154 & 2.445 \\
\hline $2 \mathrm{H}-4,41$ & 12.11 & 0.5185 & 0.787 & 2.573 \\
\hline $2 \mathrm{H}-4,82$ & 12.52 & 0.5306 & 0.317 & 2.224 \\
\hline $2 \mathrm{H}-4,132$ & 13.02 & 0.5454 & 1.120 & 2.822 \\
\hline $2 \mathrm{H}-5,41$ & 13.61 & 0.5628 & 1.017 & 2.365 \\
\hline $2 \mathrm{H}-5,82$ & 14.02 & 0.5749 & -0.314 & 2.364 \\
\hline $2 \mathrm{H}-5,82$ & 14.02 & 0.5749 & -0.555 & 1.735 \\
\hline $2 \mathrm{H}-5,132$ & 14.52 & 0.5896 & -0.021 & 2.406 \\
\hline $2 \mathrm{H}-6,42$ & 15.12 & 0.6073 & -0.354 & 2.124 \\
\hline $2 \mathrm{H}-6,82$ & 15.52 & 0.6191 & -0.212 & 3.352 \\
\hline $2 \mathrm{H}-6,132$ & 16.02 & 0.6227 & -0.716 & 2.903 \\
\hline $2 \mathrm{H}-7,41$ & 16.61 & 0.6262 & -0.435 & 2.668 \\
\hline $2 \mathrm{H}-7,41$ & 16.61 & 0.6262 & -0.595 & 2.331 \\
\hline $3 \mathrm{H}-1,28$ & 16.98 & 0.6283 & -0.912 & 2.924 \\
\hline $3 \mathrm{H}-1,116$ & 17.86 & 0.6334 & -1.084 & 2.115 \\
\hline $3 \mathrm{H}-2,28$ & 18.48 & 0.6370 & 0.025 & 2.037 \\
\hline $3 \mathrm{H}-2,81$ & 19.01 & 0.6401 & 1.322 & 2.783 \\
\hline $3 \mathrm{H}-2,81$ & 19.01 & 0.6401 & 1.348 & 2.689 \\
\hline $3 \mathrm{H}-2,116$ & 19.36 & 0.6421 & 1.392 & 2.921 \\
\hline $3 \mathrm{H}-2,116$ & 19.36 & 0.6421 & 1.459 & 3.146 \\
\hline $3 \mathrm{H}-3,28$ & 19.98 & 0.6457 & 0.262 & 2.261 \\
\hline $3 \mathrm{H}-3,28$ & 19.98 & 0.6457 & 0.301 & 2.238 \\
\hline $3 \mathrm{H}-3,28$ & 19.98 & 0.6457 & 0.427 & 2.254 \\
\hline $3 \mathrm{H}-3,82$ & 20.52 & 0.6488 & 0.252 & 2.302 \\
\hline $3 \mathrm{H}-3,116$ & 20.86 & 0.6508 & 0.146 & 2.265 \\
\hline $3 \mathrm{H}-4,82$ & 22.02 & 0.6575 & -0.161 & 2.554 \\
\hline $3 \mathrm{H}-4,116$ & 22.36 & 0.6595 & -0.756 & 2.609 \\
\hline $3 \mathrm{H}-4,116$ & 22.36 & 0.6595 & -1.193 & 2.455 \\
\hline $3 \mathrm{H}-5,28$ & 22.98 & 0.6631 & 1.783 & 3.405 \\
\hline $3 \mathrm{H}-5,28$ & 22.98 & 0.6631 & 1.909 & 3.774 \\
\hline $3 \mathrm{H}-5,82$ & 23.52 & 0.6662 & 0.179 & 2.820 \\
\hline $3 \mathrm{H}-5,116$ & 23.86 & 0.6682 & 0.431 & 3.095 \\
\hline $3 \mathrm{H}-6,28$ & 24.48 & 0.6718 & 0.400 & 2.550 \\
\hline $3 \mathrm{H}-6,82$ & 25.02 & 0.6749 & 0.156 & 2.524 \\
\hline $3 \mathrm{H}-6,116$ & 25.36 & 0.6769 & 0.053 & 2.561 \\
\hline $5 \mathrm{H}-1,21$ & 35.91 & 0.7650 & 2.396 & 3.354 \\
\hline $5 \mathrm{H}-1,21$ & 35.91 & 0.7650 & 2.328 & 3.443 \\
\hline $5 \mathrm{H}-1,51$ & 36.21 & 0.7725 & 1.030 & 2.953 \\
\hline $5 \mathrm{H}-1,51$ & 36.21 & 0.7725 & 1.080 & 3.006 \\
\hline $5 \mathrm{H}-1,81$ & 36.51 & 0.7801 & 3.226 & 3.668 \\
\hline $5 \mathrm{H}-1,81$ & 36.51 & 0.7801 & 3.121 & 3.551 \\
\hline $5 \mathrm{H}-2,21$ & 37.41 & 0.8026 & 1.103 & 2.893 \\
\hline $5 \mathrm{H}-2,51$ & 37.71 & 0.8101 & 0.657 & 2.518 \\
\hline $5 \mathrm{H}-2,81$ & 38.01 & 0.8176 & 0.891 & 2.702 \\
\hline $5 \mathrm{H}-3,21$ & 38.91 & 0.8401 & 0.671 & 2.649 \\
\hline
\end{tabular}

Table 3 (continued).

\begin{tabular}{|c|c|c|c|c|}
\hline $\begin{array}{l}\text { Core, section, } \\
\text { interval }(\mathrm{cm})\end{array}$ & $\begin{array}{l}\text { Depth } \\
\text { (mbsf) }\end{array}$ & $\begin{array}{l}\text { Age } \\
\text { (Ma) }\end{array}$ & $\delta^{13} \mathrm{C}$ & $\delta^{18} \mathrm{O}$ \\
\hline $5 \mathrm{H}-3,51$ & 39.21 & 0.8476 & 0.285 & 2.417 \\
\hline $5 \mathrm{H}-3,81$ & 39.51 & 0.8551 & 0.457 & 2.316 \\
\hline $5 \mathrm{H}-4,21$ & 40.41 & 0.8777 & 0.178 & 2.462 \\
\hline $5 \mathrm{H}-4,51$ & 40.71 & 0.8852 & 0.330 & 2.444 \\
\hline $5 \mathrm{H}-4,82$ & 41.02 & 0.8929 & 0.355 & 2.781 \\
\hline $5 \mathrm{H}-5,21$ & 41.91 & 0.9152 & -0.203 & 2.480 \\
\hline $5 \mathrm{H}-5,51$ & 42.21 & 0.9227 & -0.074 & 2.485 \\
\hline $5 \mathrm{H}-5,82$ & 42.52 & 0.9305 & -0.089 & 2.649 \\
\hline $5 \mathrm{H}-5,111$ & 42.81 & 0.9377 & -0.123 & 2.662 \\
\hline $5 \mathrm{H}-5,141$ & 43.11 & 0.9452 & -0.096 & 2.367 \\
\hline $5 \mathrm{H}-6,21$ & 43.41 & 0.9527 & -0.157 & 2.358 \\
\hline $5 \mathrm{H}-6,51$ & 43.71 & 0.9602 & -0.408 & 2.538 \\
\hline $5 \mathrm{H}-6,81$ & 44.01 & 0.9677 & 0.043 & 2.606 \\
\hline $5 \mathrm{H}-6,111$ & 44.31 & 0.9753 & -0.362 & 2.465 \\
\hline $5 \mathrm{H}-6,141$ & 44.61 & 0.9817 & -0.506 & 2.543 \\
\hline $6 \mathrm{H}-1,20$ & 45.40 & 0.9943 & 0.419 & 2.370 \\
\hline $6 \mathrm{H}-1,55$ & 45.75 & 0.9998 & -0.087 & 2.015 \\
\hline $6 \mathrm{H}-1,85$ & 46.05 & 1.0046 & 0.137 & 2.185 \\
\hline $6 \mathrm{H}-1,106$ & 46.26 & 1.0079 & 0.248 & 2.349 \\
\hline $6 \mathrm{H}-1,141$ & 46.61 & 1.0135 & 0.049 & 2.161 \\
\hline $6 \mathrm{H}-2,20$ & 46.90 & 1.0180 & -0.010 & 2.119 \\
\hline $6 \mathrm{H}-2,55$ & 47.25 & 1.0236 & 0.645 & 2.510 \\
\hline $6 \mathrm{H}-2,85$ & 47.55 & 1.0283 & 1.003 & 2.754 \\
\hline $6 \mathrm{H}-2,106$ & 47.76 & 1.0317 & 0.807 & 2.614 \\
\hline $6 \mathrm{H}-2,141$ & 48.11 & 1.0372 & -0.150 & 2.299 \\
\hline $6 \mathrm{H}-3,20$ & 48.40 & 1.0418 & -0.091 & 2.159 \\
\hline $6 \mathrm{H}-3,55$ & 48.75 & 1.0474 & -0.788 & 2.103 \\
\hline $6 \mathrm{H}-3,85$ & 49.05 & 1.0521 & -0.696 & 2.783 \\
\hline $6 \mathrm{H}-3,105$ & 49.25 & 1.0553 & -0.602 & 2.878 \\
\hline $6 \mathrm{H}-4,20$ & 49.90 & 1.0656 & -0.438 & 2.891 \\
\hline $6 \mathrm{H}-4,55$ & 50.25 & 1.0711 & 0.034 & 2.676 \\
\hline $6 \mathrm{H}-4,85$ & 50.55 & 1.0759 & -0.615 & 2.777 \\
\hline $6 \mathrm{H}-4,106$ & 50.76 & 1.0792 & -0.908 & 2.788 \\
\hline $6 \mathrm{H}-4,106$ & 50.76 & 1.0792 & -0.971 & 2.677 \\
\hline $6 \mathrm{H}-5,20$ & 51.40 & 1.0894 & -1.651 & 1.747 \\
\hline $6 \mathrm{H}-5,20$ & 51.40 & 1.0894 & -1.452 & 1.735 \\
\hline $6 \mathrm{H}-5,55$ & 51.75 & 1.0949 & -1.279 & 1.581 \\
\hline $6 \mathrm{H}-5,85$ & 52.05 & 1.0997 & -0.917 & 2.815 \\
\hline $6 \mathrm{H}-5,106$ & 52.26 & 1.1030 & -0.947 & 2.846 \\
\hline $6 \mathrm{H}-5,141$ & 52.61 & 1.1086 & -0.748 & 2.436 \\
\hline $6 \mathrm{H}-6,20$ & 52.90 & 1.1132 & -1.843 & 1.942 \\
\hline $6 \mathrm{H}-6,55$ & 53.25 & 1.1187 & -1.604 & 1.739 \\
\hline $6 \mathrm{H}-6,85$ & 53.55 & 1.1235 & -1.369 & 1.847 \\
\hline $6 \mathrm{H}-6,106$ & 53.76 & 1.1268 & -1.044 & 1.928 \\
\hline $6 \mathrm{H}-6,141$ & 54.11 & 1.1323 & -1.384 & 1.969 \\
\hline $6 \mathrm{H}-7,20$ & 54.40 & 1.1369 & -1.386 & 1.682 \\
\hline $6 \mathrm{H}-7,55$ & 54.75 & 1.1425 & -1.169 & 1.770 \\
\hline $7 \mathrm{H}-1,60$ & 5.30 & 1.1512 & -1.013 & 2.380 \\
\hline $7 \mathrm{H}-1,91$ & 1 & 1.1561 & -1.021 & 2.222 \\
\hline $7 \mathrm{H}-1,120$ & 55.90 & 1.1607 & -0.814 & 2.389 \\
\hline $7 \mathrm{H}-2,11$ & 56 & 1.1672 & -0.883 & 2.619 \\
\hline $7 \mathrm{H}-2,30$ & 56.50 & 1.1702 & -0.624 & 2.924 \\
\hline $7 \mathrm{H}-2,60$ & 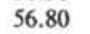 & 1.1750 & -0.550 & 3.003 \\
\hline $7 \mathrm{H}-2,91$ & .11 & 1.1799 & -0.964 & 2.260 \\
\hline $7 \mathrm{H}-2,120$ & 10 & 1.18 & 0.617 & 2.502 \\
\hline $7 \mathrm{H}-3,11$ & 57.81 & 1.1910 & 1.058 & 2.669 \\
\hline $7 \mathrm{H}-3,30$ & & 1.1940 & 0.910 & 3.081 \\
\hline $7 \mathrm{H}-3,30$ & 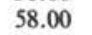 & 1.1940 & 0.665 & 2.649 \\
\hline $7 \mathrm{H}-3,60$ & 58.30 & 1.1987 & -0.682 & 2.549 \\
\hline $7 \mathrm{H}-3,91$ & 5861 & 1.2037 & -0.562 & 2.600 \\
\hline $7 \mathrm{H}-3$, & 1 & 30 & -0. & 2.797 \\
\hline $7 \mathrm{H}-3,91$ & 5861 & 1.20 & -0.602 & 2.769 \\
\hline $7 \mathrm{H}-3,120$ & 58.90 & 1.20 & -0.303 & 1.752 \\
\hline $7 \mathrm{H}-3,120$ & 58. & 1.2083 & -0.958 & 1.948 \\
\hline $7 \mathrm{H}-3,120$ & 58.9 & 1.20 & -0.7 & 2.446 \\
\hline $7 \mathrm{H}-3,120$ & 589 & 1.2083 & -1.275 & 2.176 \\
\hline $7 \mathrm{H}-4,3$ & 0 & 1.2178 & -0.480 & 2.578 \\
\hline $7 \mathrm{H}-4,30$ & 50.5 & 1.2178 & -0.461 & 2.669 \\
\hline $7 \mathrm{H}-4,60$ & 59.80 & 1.2225 & -0.632 & 2.331 \\
\hline $7 \mathrm{H}-4,91$ & & 1.2274 & 0.397 & 2.595 \\
\hline $7 \mathrm{H}-4,91$ & & 1.2274 & 0.240 & 2.797 \\
\hline $7 \mathrm{H}-4,120$ & 60.4 & 1.232 & 0.911 & 2.998 \\
\hline $7 \mathrm{H}-4,120$ & 60.4 & 1.2320 & 0.978 & 2.782 \\
\hline $7 \mathrm{H}-5,11$ & 60. & 1.23 & 1.707 & 3.275 \\
\hline 7n-J, & 60.8 & 1.2385 & 1.226 & 3.028 \\
\hline & & 1.2415 & 1.232 & 3.100 \\
\hline $7 \mathrm{H}-5,60$ & 61.30 & 1.2463 & -0.575 & 2.505 \\
\hline
\end{tabular}


Table 3 (continued).

\begin{tabular}{|c|c|c|c|c|}
\hline $\begin{array}{l}\text { Core, section, } \\
\text { interval }(\mathrm{cm})\end{array}$ & $\begin{array}{l}\text { Depth } \\
\text { (mbsf) }\end{array}$ & $\begin{array}{l}\text { Age } \\
\text { (Ma) }\end{array}$ & $\delta^{13} \mathrm{C}$ & $\delta^{18} \mathrm{O}$ \\
\hline $7 \mathrm{H}-5,91$ & 61.61 & 1.2512 & -0.490 & 2.681 \\
\hline $7 \mathrm{H}-5,120$ & 61.90 & 1.2558 & -0.534 & 2.517 \\
\hline $7 \mathrm{H}-5,120$ & 61.90 & 1.2558 & -0.398 & 2.721 \\
\hline $7 \mathrm{H}-6,11$ & 62.31 & 1.2623 & -0.975 & 2.469 \\
\hline $7 \mathrm{H}-6,11$ & 62.31 & 1.2623 & -0.562 & 2.822 \\
\hline $7 \mathrm{H}-6,30$ & 62.50 & 1.2653 & -1.506 & 2.417 \\
\hline $7 \mathrm{H}-6,30$ & 62.50 & 1.2653 & -1.549 & 2.418 \\
\hline $7 \mathrm{H}-6,60$ & 62.80 & 1.2701 & -1.409 & 2.433 \\
\hline $7 \mathrm{H}-6,60$ & 62.80 & 1.2701 & -1.607 & 2.408 \\
\hline $7 \mathrm{H}-6,90$ & 63.10 & 1.2748 & -1.055 & 2.431 \\
\hline $8 \mathrm{H}-1,30$ & 64.50 & 1.2970 & 1.145 & 2.889 \\
\hline $8 \mathrm{H}-1,81$ & 65.01 & 1.3051 & 0.118 & 2.374 \\
\hline $8 \mathrm{H}-1,111$ & 65.31 & 1.3099 & 0.496 & 2.490 \\
\hline $8 \mathrm{H}-1,140$ & 65.60 & 1.3145 & -0.214 & 2.526 \\
\hline $8 \mathrm{H}-1,140$ & 65.60 & 1.3145 & -0.299 & 2.440 \\
\hline $8 \mathrm{H}-2,30$ & 66.00 & 1.3208 & -0.592 & 2.444 \\
\hline $8 \mathrm{H}-2,51$ & 66.21 & 1.3241 & -0.608 & 2.324 \\
\hline $8 \mathrm{H}-2,81$ & 66.51 & 1.3289 & -0.682 & 2.149 \\
\hline $8 \mathrm{H}-2,81$ & 66.51 & 1.3289 & -0.665 & 1.998 \\
\hline $8 \mathrm{H}-2,111$ & 66.81 & 1.3336 & -0.998 & 1.907 \\
\hline $8 \mathrm{H}-2,140$ & 67.10 & 1.3382 & 0.023 & 2.226 \\
\hline $8 \mathrm{H}-3,31$ & 67.51 & 1.3447 & 0.958 & 2.487 \\
\hline $8 \mathrm{H}-3,48$ & 67.68 & 1.3474 & 0.420 & 2.337 \\
\hline $8 \mathrm{H}-3,82$ & 68.02 & 1.3528 & -0.148 & 2.505 \\
\hline $8 \mathrm{H}-3,82$ & 68.02 & 1.3528 & -0.141 & 2.910 \\
\hline $8 \mathrm{H}-3,111$ & 68.31 & 1.3574 & -0.319 & 2.675 \\
\hline $8 \mathrm{H}-3,140$ & 68.60 & 1.3620 & 0.094 & 2.702 \\
\hline $8 \mathrm{H}-4,31$ & 69.01 & 1.3685 & -0.339 & 2.162 \\
\hline $8 \mathrm{H}-4,31$ & 69.01 & 1.3685 & -0.332 & 2.139 \\
\hline $8 \mathrm{H}-4,51$ & 69.21 & 1.3717 & -0.012 & 2.223 \\
\hline $8 \mathrm{H}-4,81$ & 69.51 & 1.3764 & -0.793 & 2.318 \\
\hline $8 \mathrm{H}-4,81$ & 69.51 & 1.3764 & -0.647 & 2.544 \\
\hline $8 \mathrm{H}-4,111$ & 69.81 & 1.3812 & -0.563 & 2.675 \\
\hline $8 \mathrm{H}-4,140$ & 70.10 & 1.3858 & 0.066 & 2.708 \\
\hline $8 \mathrm{H}-5,31$ & 70.51 & 1.3923 & -0.439 & 2.780 \\
\hline $8 \mathrm{H}-5,51$ & 70.71 & 1.3955 & -0.388 & 2.602 \\
\hline $8 \mathrm{H}-5,81$ & 71.01 & 1.4002 & -0.379 & 2.512 \\
\hline $8 \mathrm{H}-5,81$ & 71.01 & 1.4002 & -0.709 & 2.432 \\
\hline $8 \mathrm{H}-5,111$ & 71.31 & 1.4050 & -0.606 & 2.419 \\
\hline $8 \mathrm{H}-5,140$ & 71.60 & 1.4096 & -0.826 & 2.081 \\
\hline $8 \mathrm{H}-6,31$ & 72.01 & 1.4161 & -0.525 & 2,437 \\
\hline $8 \mathrm{H}-6,51$ & 72.21 & 1.4192 & -0.413 & 2.301 \\
\hline $8 \mathrm{H}-6,81$ & 72.51 & 1.4240 & -0.326 & 2.031 \\
\hline $8 \mathrm{H}-6,111$ & 72.81 & 1.4287 & -0.552 & 2.183 \\
\hline $9 \mathrm{H}-1,51$ & 74.21 & 1.4509 & -0.064 & 2.237 \\
\hline $9 \mathrm{H}-1,51$ & 74.21 & 1.4509 & -0.012 & 2.436 \\
\hline $9 \mathrm{H}-1,81$ & 74.51 & 1.4557 & 0.069 & 2.093 \\
\hline $9 \mathrm{H}-1,111$ & 74.81 & 1.4604 & 0.313 & 2.448 \\
\hline $9 \mathrm{H}-1,141$ & 75.11 & 1.4652 & 0.155 & 2.087 \\
\hline $9 \mathrm{H}-2,21$ & 75.41 & 1.4700 & 0.242 & 2.257 \\
\hline $9 \mathrm{H}-2,51$ & 75.71 & 1.4747 & 0.046 & 2.396 \\
\hline $9 \mathrm{H}-2,81$ & 76.01 & 1.4795 & -0.092 & 2.606 \\
\hline $9 \mathrm{H}-2,111$ & 76.31 & 1.4842 & 0.001 & 2.491 \\
\hline $9 \mathrm{H}-2,141$ & 76.61 & 1.4890 & 0.013 & 2.438 \\
\hline $9 \mathrm{H}-3,21$ & 76.91 & 1.4937 & 0.012 & 2.472 \\
\hline $9 \mathrm{H}-3,51$ & 77.21 & 1.4985 & 0.197 & 2.600 \\
\hline $9 \mathrm{H}-3,81$ & 77.51 & 1.5032 & 0.046 & 2.489 \\
\hline $9 \mathrm{H}-3,111$ & 77.81 & 1.5080 & 0.050 & 2.378 \\
\hline $9 \mathrm{H}-3,141$ & 78.11 & 1.5128 & 0.178 & 2.615 \\
\hline $9 \mathrm{H}-4,21$ & 78.41 & 1.5175 & 0.415 & 2.688 \\
\hline $9 \mathrm{H}-4,51$ & 78.71 & 1.5223 & 0.493 & 2.615 \\
\hline $9 \mathrm{H}-4,81$ & 79.01 & 1.5270 & 0.445 & 2.397 \\
\hline $9 \mathrm{H}-4,81$ & 79.01 & 1.5270 & 0.376 & 2.373 \\
\hline $9 \mathrm{H}-5,21$ & 79.91 & 1.5413 & -0.296 & 2.732 \\
\hline $9 \mathrm{H}-7,21$ & 82.91 & 1.5888 & -0.693 & 2.640 \\
\hline $9 \mathrm{H}-7,21$ & 82.91 & 1.5888 & -0.981 & 2.519 \\
\hline $10 \mathrm{H}-1,20$ & 83.40 & 1.5966 & -0.183 & 2.652 \\
\hline $10 \mathrm{H}-1,20$ & 83.40 & 1.5966 & -0.264 & 2.077 \\
\hline $10 \mathrm{H}-1,20$ & 83.40 & 1.5966 & -0.492 & 2.182 \\
\hline $10 \mathrm{H}-1,20$ & 83.40 & 1.5966 & -0.186 & 2.527 \\
\hline $10 \mathrm{H}-2,20$ & 84.90 & 1.6204 & -1.184 & 2.302 \\
\hline $10 \mathrm{H}-3,20$ & 86.40 & 1.6442 & -1.504 & 2.026 \\
\hline $10 \mathrm{H}-4,20$ & 87.90 & 1.6690 & -0.794 & 2.552 \\
\hline $10 \mathrm{H}-4,20$ & 87.90 & 1.6690 & -0.686 & 2.536 \\
\hline $10 \mathrm{H}-5,20$ & 89.40 & 1.6958 & 0.309 & 2.400 \\
\hline $10 \mathrm{H}-5,20$ & 89.40 & 1.6958 & 0.290 & 2.362 \\
\hline $10 \mathrm{H}-6,20$ & 90.90 & 1.7227 & -0.172 & 2.165 \\
\hline
\end{tabular}

Table 3 (continued).

\begin{tabular}{|c|c|c|c|c|}
\hline $\begin{array}{l}\text { Core, section, } \\
\text { interval }(\mathrm{cm})\end{array}$ & $\begin{array}{l}\text { Depth } \\
\text { (mbsf) }\end{array}$ & $\begin{array}{l}\text { Age } \\
\text { (Ma) }\end{array}$ & $\delta^{13} \mathrm{C}$ & $\delta^{18} \mathrm{O}$ \\
\hline $10 \mathrm{H}-7,20$ & 92.40 & 1.7495 & -0.581 & 2.491 \\
\hline $11 \mathrm{H}-1,21$ & 92.91 & 1.7586 & -0.431 & 2.208 \\
\hline $11 \mathrm{H}-1,21$ & 92.91 & 1.7586 & -0.157 & 2.107 \\
\hline $11 \mathrm{H}-2,21$ & 94.41 & 1.7855 & -0.108 & 2.265 \\
\hline $11 \mathrm{H}-3,21$ & 95.91 & 1.8123 & -0.556 & 2.446 \\
\hline $11 \mathrm{H}-3,21$ & 95.91 & 1.8123 & -1.341 & 2.144 \\
\hline $11 \mathrm{H}-3,21$ & 95.91 & 1.8123 & -0.784 & 2.357 \\
\hline $11 \mathrm{H}-3,21$ & 95.91 & 1.8123 & -1.516 & 2.165 \\
\hline $11 \mathrm{H}-3,21$ & 95.91 & 1.8123 & -0.740 & 2.339 \\
\hline $11 \mathrm{H}-4,21$ & 97.41 & 1.8392 & -0.894 & 2.524 \\
\hline $11 \mathrm{H}-4,21$ & 97.41 & 1.8392 & -0.885 & 2.447 \\
\hline $11 \mathrm{H}-5,21$ & 98.91 & 1.8660 & -0.440 & 2.260 \\
\hline $11 \mathrm{H}-5,21$ & 98.91 & 1.8660 & -0.430 & 2.238 \\
\hline $11 \mathrm{H}-5,21$ & 98.91 & 1.8660 & -0.605 & 2.366 \\
\hline $11 \mathrm{H}-6,21$ & 100.41 & 1.8913 & -0.155 & 2.273 \\
\hline $11 \mathrm{H}-6,21$ & 100.41 & 1.8913 & -0.181 & 2.284 \\
\hline $12 \mathrm{H}-1,21$ & 102.41 & 1.9073 & -0.315 & 2.331 \\
\hline $12 \mathrm{H}-1,21$ & 102.41 & 1.9073 & -0.250 & 2.298 \\
\hline $12 \mathrm{H}-1,21$ & 102.41 & 1.9073 & -0.263 & 2.318 \\
\hline $12 \mathrm{H}-2,21$ & 103.91 & 1.9193 & 0.210 & 2.725 \\
\hline $12 \mathrm{H}-2,21$ & 103.91 & 1.9193 & 0.197 & 2.715 \\
\hline $12 \mathrm{H}-3,21$ & 105.41 & 1.9313 & -0.220 & 2.331 \\
\hline $12 \mathrm{H}-3,21$ & 105.41 & 1.9313 & -0.206 & 2.309 \\
\hline $12 \mathrm{H}-3,21$ & 105.41 & 1.9313 & -0.332 & 2.253 \\
\hline $12 \mathrm{H}-4,21$ & 106.91 & 1.9433 & -0.357 & 2.787 \\
\hline $12 \mathrm{H}-4,21$ & 106.91 & 1.9433 & -0.348 & 2.720 \\
\hline $12 \mathrm{H}-4,21$ & 106.91 & 1.9433 & -0.527 & 2.320 \\
\hline $12 \mathrm{H}-5,21$ & 108.41 & 1.9553 & 0.392 & 2.189 \\
\hline $12 \mathrm{H}-5,21$ & 108.41 & 1.9553 & 0.434 & 2.420 \\
\hline $12 \mathrm{H}-5,21$ & 108.41 & 1.9553 & 0.381 & 2.515 \\
\hline $12 \mathrm{H}-6,21$ & 109.91 & 1.9673 & -0.064 & 2.899 \\
\hline $13 \mathrm{H}-1,21$ & 111.91 & 1.9833 & -0.420 & 2.476 \\
\hline $13 \mathrm{H}-2,21$ & 113.41 & 1.9953 & -0.390 & 2.271 \\
\hline $13 \mathrm{H}-3,21$ & 114.91 & 2.0073 & -0.429 & 2.795 \\
\hline $13 \mathrm{H}-4,21$ & 116.41 & 2.0193 & -0.330 & 2.422 \\
\hline $13 \mathrm{H}-5,21$ & 117.91 & 2.0313 & -0.205 & 2.730 \\
\hline $13 \mathrm{H}-6,21$ & 119.41 & 2.0433 & -0.077 & 2.662 \\
\hline $13 \mathrm{H}-7,21$ & 120.91 & 2.0553 & 0.148 & 2.239 \\
\hline $13 \mathrm{H}-7,21$ & 120.91 & 2.0553 & 0.415 & 2.514 \\
\hline $14 \mathrm{H}-1,21$ & 121.41 & 2.0593 & -0.240 & 2.509 \\
\hline $14 \mathrm{H}-2,21$ & 122.91 & 2.0713 & -0.423 & 2.517 \\
\hline $14 \mathrm{H}-3,21$ & 124.41 & 2.0833 & -0.504 & 2.292 \\
\hline $14 \mathrm{H}-4,21$ & 125.91 & 2.0953 & 0.312 & 2.754 \\
\hline $14 \mathrm{H}-5,21$ & 127.41 & 2.1073 & 0.015 & 2.762 \\
\hline $14 \mathrm{H}-6,21$ & 128.91 & 2.1193 & -0.341 & 2.427 \\
\hline $15 \mathrm{H}-1,21$ & 130.91 & 2.1353 & -0.205 & 1.897 \\
\hline $15 \mathrm{H}-1,21$ & 130.91 & 2.1353 & -0.152 & 2.001 \\
\hline $15 \mathrm{H}-1,21$ & 130.91 & 2.1353 & -0.726 & 1.605 \\
\hline $15 \mathrm{H}-2,21$ & 132.41 & 2.1481 & -0.227 & 2.217 \\
\hline $15 \mathrm{H}-2,21$ & 132.41 & 2.1481 & -0.340 & 2.227 \\
\hline $15 \mathrm{H}-3,21$ & 133.91 & 2.1614 & -0.114 & 2.126 \\
\hline $15 \mathrm{H}-3,141$ & 135.11 & 2.1720 & -0.197 & 2.085 \\
\hline $15 \mathrm{H}-5,21$ & 137.21 & 2.1906 & -0.580 & 2.461 \\
\hline $15 \mathrm{H}-6,21$ & 138.41 & 2.2013 & -0.014 & 2.158 \\
\hline $15 \mathrm{H}-7,21$ & 139.91 & 2.2146 & -0.627 & 2.026 \\
\hline $16 \mathrm{H}-1,21$ & 140.41 & 2.2190 & -0.943 & 1.730 \\
\hline $16 \mathrm{H}-2,21$ & 141.91 & 2.2323 & -0.636 & 2.223 \\
\hline $16 \mathrm{H}-3,21$ & 143.41 & 2.2456 & -0.723 & 2.023 \\
\hline $16 \mathrm{H}-4,21$ & 144.91 & 2.2589 & -0.114 & 2.823 \\
\hline $16 \mathrm{H}-5,21$ & 146.41 & 2.2722 & 0.006 & 2.336 \\
\hline $16 \mathrm{H}-6,21$ & 147.91 & 2.2855 & -0.423 & 2.269 \\
\hline $17 \mathrm{X}-1,21$ & 149.91 & 2.3032 & -0.477 & 1.952 \\
\hline $17 \mathrm{X}-2,21$ & 151.41 & 2.3165 & -0.068 & 2.829 \\
\hline $17 X-3,21$ & 152.91 & 2.3298 & -0.250 & 2.509 \\
\hline $17 X-3,21$ & 152.91 & 2.3298 & -0.276 & 2.318 \\
\hline $17 X-4,21$ & 154.41 & 2.3431 & -0.847 & 2.039 \\
\hline $17 X-5,21$ & 155.91 & 2.3564 & -0.537 & 2.620 \\
\hline $17 X-6,21$ & 157.41 & 2.3697 & -0.594 & 2.364 \\
\hline $18 \mathrm{X}-1,21$ & 159.41 & 2.3875 & -0.044 & 2.345 \\
\hline $18 \mathrm{X}-2,21$ & 160.91 & 2.4008 & 0.081 & 2.337 \\
\hline $18 X-3,21$ & 162.41 & 2.4141 & -0.017 & 2.587 \\
\hline $18 X-4,21$ & 163.91 & 2.4274 & -0.157 & 2.739 \\
\hline $18 X-5,21$ & 165.41 & 2.4407 & 0.025 & 2.108 \\
\hline $18 X-6,21$ & 166.91 & 2.4540 & -0.420 & 2.532 \\
\hline $19 X-1,21$ & 168.91 & 2.5488 & -0.092 & 2.893 \\
\hline $19 X-2,21$ & 170.41 & 2.6873 & 0.830 & 2.589 \\
\hline $19 X-3,21$ & 171.91 & 2.7428 & 0.955 & 2.758 \\
\hline
\end{tabular}


Table 3 (continued).

\begin{tabular}{|c|c|c|c|c|}
\hline $\begin{array}{l}\text { Core, section, } \\
\text { interval }(\mathrm{cm})\end{array}$ & $\begin{array}{l}\text { Depth } \\
\text { (mbsf) }\end{array}$ & $\begin{array}{l}\text { Age } \\
(\mathrm{Ma})\end{array}$ & $\delta^{13} \mathrm{C}$ & $\delta^{18} \mathrm{O}$ \\
\hline $19 X-4,21$ & 173.41 & 2.7983 & 0.466 & 2.454 \\
\hline $19 X-4,21$ & 173.41 & 2.7983 & 0.428 & 2.529 \\
\hline $19 X-5,21$ & 174.91 & 2.8538 & 1.783 & 3.470 \\
\hline $19 X-6,21$ & 176.41 & 2.9093 & 1.783 & 3.372 \\
\hline $20 X-1,21$ & 178.41 & 3.0295 & 1.221 & 2.998 \\
\hline $20 X-2,21$ & 179.91 & 3.1238 & 0.508 & 2.358 \\
\hline $20 \times-2,21$ & 179.91 & 3.1238 & 0.544 & 2.439 \\
\hline $20 \mathrm{X}-3,21$ & 181.41 & 3.1911 & 2.247 & 3.576 \\
\hline $20 \times-3,21$ & 181.41 & 3.1911 & 2.177 & 3.343 \\
\hline $20 X-4,21$ & 182.91 & 3.2504 & 1.493 & 2.966 \\
\hline $20 X-5,21$ & 184.41 & 3.3098 & 2.002 & 2.745 \\
\hline $20 \mathrm{X}-6,21$ & 185.91 & 3.3691 & 0.515 & 2.156 \\
\hline $20 \mathrm{X}-6,21$ & 185.91 & 3.3691 & 0.598 & 2.104 \\
\hline $20 X-6,21$ & 185.91 & 3.3691 & 0.661 & 1.734 \\
\hline $20 \mathrm{X}-7,21$ & 187.41 & 3.4380 & 0.190 & 2.044 \\
\hline $20 X-7,21$ & 187.41 & 3.4380 & 1.095 & 2.875 \\
\hline $21 X-1,46$ & 188.16 & 3.4775 & 0.422 & 2.112 \\
\hline $21 X-1,51$ & 188.21 & 3.4802 & 0.228 & 2.038 \\
\hline $21 X-2,46$ & 189.66 & 3.5567 & 0.436 & 2.059 \\
\hline $21 X-2,46$ & 189.66 & 3.5567 & 0.438 & 2.090 \\
\hline $21 X-3,46$ & 191.16 & 3.6358 & 0.424 & 1.911 \\
\hline $22 \mathrm{X}-1,21$ & 197.41 & 3.9037 & 0.599 & 2.081 \\
\hline $22 X-2,21$ & 198.91 & 3.9257 & 0.759 & 2.243 \\
\hline $22 X-2,21$ & 198.91 & 3.9257 & 0.730 & 2.227 \\
\hline $22 \mathrm{X}-3,21$ & 200.41 & 3.9476 & 1.576 & 2.222 \\
\hline $22 \mathrm{X}-3,51$ & 200.71 & 3.9520 & 1.661 & 2.651 \\
\hline $22 X-4,21$ & 201.91 & 3.9696 & 1.663 & 2.688 \\
\hline $22 X-4,51$ & 202.21 & 3.9856 & 1.084 & 2.617 \\
\hline $22 X-5,21$ & 203.41 & 4.0549 & 2.576 & 2.740 \\
\hline $22 X-5,51$ & 203.71 & 4.0723 & 2.590 & 3.198 \\
\hline $22 X-5,140$ & 204.60 & 4.1094 & 1.656 & 2.482 \\
\hline $23 \mathrm{X}-1,21$ & 206.91 & 4.1624 & 0.550 & 2.118 \\
\hline $23 \times-1,51$ & 207.21 & 4.1693 & 0.464 & 2.026 \\
\hline $23 \mathrm{X}-2,21$ & 208.41 & 4.1969 & 0.463 & 2.128 \\
\hline $23 X-3,21$ & 209.91 & 4.2313 & 0.370 & 2.072 \\
\hline $23 \mathrm{X}-3,51$ & 210.21 & 4.2382 & 0.326 & 1.841 \\
\hline $23 \mathrm{X}-4,21$ & 211.41 & 4.3454 & 0.816 & 2.278 \\
\hline $23 X-4,51$ & 211.71 & 4.3737 & 1.049 & 2.332 \\
\hline $23 X-5,21$ & 212.91 & 4.4524 & 0.672 & 2.286 \\
\hline $23 X-6,21$ & 214.41 & 4.4992 & 1.448 & 2.485 \\
\hline $24 X-1,21$ & 216.41 & 4.5484 & 0.990 & 2.241 \\
\hline $24 X-2,21$ & 217.91 & 4.6277 & 0.659 & 2.113 \\
\hline $24 X-3,21$ & 219.41 & 4.7672 & 0.808 & 1.996 \\
\hline $24 X-3,51$ & 219.71 & 4.7893 & 0.963 & 2.062 \\
\hline $24 X-4,51$ & 221.21 & 4.8967 & 2.010 & 2.904 \\
\hline $24 X-5,21$ & 222.41 & 4.9825 & 0.583 & 2.417 \\
\hline $24 X-5,51$ & 222.71 & 5.0040 & 1.276 & 2.388 \\
\hline $24 X-6,21$ & 223.91 & 5.0899 & 1.065 & 2.318 \\
\hline $25 \mathrm{X}-1,21$ & 225.91 & 5.3801 & 1.658 & 2.302 \\
\hline $25 X-2,21$ & 227.41 & 5.4242 & 1.234 & 2.048 \\
\hline $25 X-2,48$ & 227.68 & 5.4322 & 1.149 & 1.991 \\
\hline $25 \mathrm{X}-3,21$ & 228.91 & 5.4684 & 1.711 & 2.335 \\
\hline $25 X-4,21$ & 230.41 & 5.5126 & 1.928 & 2.822 \\
\hline $25 X-6,21$ & 233.41 & 5.6505 & 1.769 & 2.605 \\
\hline $114-704 \mathrm{~B}-$ & & & & \\
\hline $1 \mathrm{H}-1,31$ & 0.31 & 0.0530 & 0.031 & 2.365 \\
\hline $1 \mathrm{H}-1,61$ & 0.61 & 0.1043 & -0.002 & 2.338 \\
\hline $1 \mathrm{H}-1,90$ & 0.90 & 0.1540 & 0.254 & 2.378 \\
\hline $1 \mathrm{H}-1,121$ & 1.21 & 0.1971 & 0.721 & 2.598 \\
\hline $1 \mathrm{H}-2,31$ & 1.81 & 0.2148 & 0.493 & 1.861 \\
\hline $1 \mathrm{H}-2,61$ & 2.11 & 0.2236 & -0.082 & 1.514 \\
\hline $1 \mathrm{H}-2,90$ & 2.40 & 0.2322 & 0.151 & 1.361 \\
\hline $1 \mathrm{H}-2,121$ & 2.71 & 0.2413 & 0.186 & 1.593 \\
\hline $1 \mathrm{H}-3,45$ & 3.45 & 0.2631 & -0.629 & 1.740 \\
\hline $1 \mathrm{H}-3,102$ & 4.02 & 0.2799 & 0.225 & 2.110 \\
\hline $1 \mathrm{H}-4,142$ & 5.92 & 0.3360 & 2.988 & 3.799 \\
\hline $1 \mathrm{H}-5,20$ & 6.20 & 0.3442 & 3.088 & 3.005 \\
\hline $1 \mathrm{H}-5,45$ & 6.45 & 0.3516 & 2.637 & 3.062 \\
\hline $2 \mathrm{H}-\mathrm{I}, 8$ & 6.78 & 0.3613 & 2.695 & 3.199 \\
\hline $2 \mathrm{H}-1,43$ & 7.13 & 0.3717 & 1.504 & 2.835 \\
\hline $2 \mathrm{H}-1,108$ & 7.78 & 0.3908 & 2.999 & 3.415 \\
\hline $2 \mathrm{H}-1,143$ & 8.13 & 0.4012 & 1.497 & 3.237 \\
\hline $2 \mathrm{H}-2,61$ & 8.81 & 0.4212 & 1.780 & 3.052 \\
\hline $2 \mathrm{H}-2,122$ & 9.42 & 0.4392 & 1.345 & 2.704 \\
\hline $2 \mathrm{H}-3,18$ & 9.88 & 0.4528 & 1.343 & 2.680 \\
\hline $2 \mathrm{H}-3,62$ & 10.32 & 0.4658 & 0.106 & 2.730 \\
\hline
\end{tabular}

Table 3 (continued).

\begin{tabular}{|c|c|c|c|c|}
\hline $\begin{array}{l}\text { Core, section, } \\
\text { interval }(\mathrm{cm})\end{array}$ & $\begin{array}{l}\text { Depth } \\
\text { (mbsf) }\end{array}$ & $\begin{array}{l}\text { Age } \\
\text { (Ma) }\end{array}$ & $\delta^{13} \mathrm{C}$ & $\delta^{18} \mathrm{O}$ \\
\hline $2 \mathrm{H}-3,103$ & 10.73 & 0.4778 & -0.132 & 2.459 \\
\hline $2 \mathrm{H}-3,140$ & 11.10 & 0.4888 & -0.089 & 2.214 \\
\hline $2 \mathrm{H}-4,17$ & 11.37 & 0.4967 & 0.144 & 2.358 \\
\hline $2 \mathrm{H}-4,58$ & 11.78 & 0.5088 & -0.332 & 1.301 \\
\hline $2 \mathrm{H}-4,99$ & 12.19 & 0.5209 & -0.200 & 2.252 \\
\hline $2 \mathrm{H}-4,99$ & 12.19 & 0.5209 & -0.599 & 1.893 \\
\hline $2 \mathrm{H}-4,99$ & 12.19 & 0.5209 & -0.600 & 1.971 \\
\hline $2 \mathrm{H}-4,138$ & 12.58 & 0.5324 & -1.029 & 2.534 \\
\hline $2 \mathrm{H}-5,15$ & 12.85 & 0.5404 & -0.844 & 2.386 \\
\hline $2 \mathrm{H}-5,55$ & 13.25 & 0.5522 & -1.154 & 2.429 \\
\hline $2 \mathrm{H}-5,97$ & 13.67 & 0.5646 & -1.156 & 2.719 \\
\hline $2 \mathrm{H}-5,137$ & 14.07 & 0.5764 & -0.894 & 2.268 \\
\hline $3 \mathrm{H}-1,21$ & 16.41 & 0.6250 & -1.367 & 2.486 \\
\hline $3 \mathrm{H}-1,61$ & 16.81 & 0.6273 & -0.886 & 2.644 \\
\hline $3 \mathrm{H}-1,95$ & 17.15 & 0.6293 & -0.598 & 2.765 \\
\hline $3 \mathrm{H}-1,141$ & 17.61 & 0.6320 & -0.765 & 2.893 \\
\hline $3 \mathrm{H}-2,21$ & 17.91 & 0.6337 & -2.165 & 2.209 \\
\hline $3 \mathrm{H}-2,21$ & 17.91 & 0.6337 & -2.299 & 1.553 \\
\hline $3 \mathrm{H}-2,61$ & 18.31 & 0.6360 & -0.664 & 1.998 \\
\hline $3 \mathrm{H}-2,95$ & 18.65 & 0.6380 & 0.370 & 2.368 \\
\hline $3 \mathrm{H}-2,14 \mathrm{I}$ & 19.11 & 0.6407 & 0.248 & 2.272 \\
\hline $3 \mathrm{H}-3,21$ & 19.41 & 0.6424 & 0.336 & 2.304 \\
\hline $3 \mathrm{H}-3,21$ & 19.41 & 0.6424 & -0.361 & 2.551 \\
\hline $3 \mathrm{H}-3,61$ & 19.81 & 0.6447 & 1.446 & 2.593 \\
\hline $3 \mathrm{H}-3,95$ & 20.15 & 0.6467 & 1.648 & 2.473 \\
\hline $3 \mathrm{H}-3,141$ & 20.61 & 0.6494 & -0.542 & 2.680 \\
\hline $3 \mathrm{H}-4,21$ & 20.91 & 0.6511 & -0.268 & 2.809 \\
\hline $3 \mathrm{H}-4,61$ & 21.31 & 0.6534 & -0.351 & 2.821 \\
\hline $3 \mathrm{H}-4,95$ & 21.65 & 0.6554 & -0.447 & 2.798 \\
\hline $3 \mathrm{H}-4,141$ & 22.11 & 0.6581 & -0.397 & 2.755 \\
\hline $3 \mathrm{H}-5,2 \mathrm{I}$ & 22.41 & 0.6598 & 0.586 & 2.398 \\
\hline $3 \mathrm{H}-5,61$ & 22.81 & 0.6621 & -1.122 & 1.965 \\
\hline $3 \mathrm{H}-5,95$ & 23.15 & 0.6641 & -1.511 & 2.373 \\
\hline $3 \mathrm{H}-5,141$ & 23.61 & 0.6668 & -0.307 & 2.865 \\
\hline $3 \mathrm{H}-6,21$ & 23.91 & 0.6685 & -0.845 & 1.944 \\
\hline $3 \mathrm{H}-6,21$ & 23.91 & 0.66 & -1.2 & 1.673 \\
\hline $3 \mathrm{H}-6,61$ & 24.31 & & -1.634 & 2.280 \\
\hline $3 \mathrm{H}-6,95$ & 24.65 & 0.6728 & -0.252 & 2.088 \\
\hline $3 \mathrm{H}-6,141$ & 25.11 & 0.6755 & -0.458 & 2.330 \\
\hline $3 \mathrm{H}-7,21$ & 25.41 & 0.6772 & -0.840 & 2.500 \\
\hline $3 \mathrm{H}-7,61$ & 25.81 & 0.6795 & -0.185 & 2.616 \\
\hline $4 \mathrm{H}-1,31$ & 26.01 & 0.6807 & -1.108 & 2.046 \\
\hline $4 \mathrm{H}-1,71$ & 26.41 & 0.6830 & -1.654 & 2.149 \\
\hline $4 \mathrm{H}-1,106$ & 26.76 & 0.6850 & -0.718 & 2.121 \\
\hline $4 \mathrm{H}-1,121$ & 26.91 & 0.6859 & -1.308 & 2.731 \\
\hline $4 \mathrm{H}-1,146$ & 27.16 & 0.6874 & -0.532 & 2.969 \\
\hline $4 \mathrm{H}-2,31$ & 27.51 & 0.6894 & 0.045 & 2.609 \\
\hline $4 \mathrm{H}-2,71$ & 27.91 & 0.6917 & -0.493 & 2.446 \\
\hline $4 \mathrm{H}-2,106$ & 28.26 & 0.6937 & -0.802 & 2.687 \\
\hline $4 \mathrm{H}-2,121$ & 28.41 & 0.6946 & -0.710 & 2.430 \\
\hline $4 \mathrm{H}-2,146$ & 28.66 & 0.6961 & -0.921 & 2.906 \\
\hline $4 \mathrm{H}-2,146$ & 28.66 & 0.6961 & -2.289 & 1.182 \\
\hline $4 \mathrm{H}-3,31$ & 29.01 & 0.6981 & 0.360 & 2.486 \\
\hline $4 \mathrm{H}-3,71$ & 29.41 & 0.7004 & 0.376 & 2.186 \\
\hline $4 \mathrm{H}-3,106$ & 29.76 & 0.7024 & -1.511 & 1.659 \\
\hline $4 \mathrm{H}-3,121$ & 29.91 & 0.7 & -0. & 1.960 \\
\hline $4 \mathrm{H}-3,146$ & 30.16 & 0.7048 & - & 1.978 \\
\hline $4 \mathrm{H}-4,31$ & 30.51 & 0.7068 & -0. & 2.201 \\
\hline $4 \mathrm{H}-4,71$ & 30.91 & 0.7091 & -0.624 & 2.157 \\
\hline $4 \mathrm{H}-4,106$ & 31.26 & 0.7111 & -0.781 & 2.081 \\
\hline $4 \mathrm{H}-4,146$ & 31.60 & 0.7131 & -0.337 & 2.326 \\
\hline $4 \mathrm{H}-5,31$ & 32.01 & 0.7155 & -0.781 & 2.710 \\
\hline $4 \mathrm{H}-5,40$ & 32.10 & 0.7160 & 0.711 & 2.793 \\
\hline $4 \mathrm{H}-5,71$ & 32.41 & 0.7178 & -0.983 & 2.399 \\
\hline $4 \mathrm{H}-5,106$ & 32.76 & 0.7199 & -1.108 & 2.421 \\
\hline $4 \mathrm{H}-5,121$ & 32.91 & 0.7207 & -0.870 & 3.947 \\
\hline $4 \mathrm{H}-5,146$ & 33.16 & 0.7222 & -0.6 & 2.591 \\
\hline $4 \mathrm{H}-6,31$ & 33.51 & 0.7242 & -0.486 & 2.432 \\
\hline $4 \mathrm{H}-6,71$ & 33.91 & 0.7265 & -0.186 & 2.328 \\
\hline $4 \mathrm{H}-6,106$ & 34.26 & 0.7286 & 1.340 & 3.085 \\
\hline $24 \mathrm{X}-1,21$ & 213.91 & 4.4870 & 0.700 & 2.449 \\
\hline $24 X-1,51$ & 214.21 & 4.4943 & 0.786 & 1.736 \\
\hline $24 \mathrm{X}-1,81$ & 214.51 & 4.5017 & 1.288 & 2.434 \\
\hline $24 X-1,111$ & 214.81 & 4.5091 & 0.628 & 2.281 \\
\hline $24 \mathrm{X}-1,141$ & 215.11 & 4.5164 & 0.918 & 2.057 \\
\hline $24 X-2,21$ & 215.41 & 4.5238 & 0.470 & 2.007 \\
\hline $24 X-2,51$ & 215.71 & 4.5312 & 0.505 & 1.975 \\
\hline
\end{tabular}


Table 3 (continued).

\begin{tabular}{|c|c|c|c|c|}
\hline $\begin{array}{l}\text { Core, section, } \\
\text { interval }(\mathrm{cm})\end{array}$ & $\begin{array}{l}\text { Depth } \\
\text { (mbsf) }\end{array}$ & $\begin{array}{l}\text { Age } \\
(\mathrm{Ma})\end{array}$ & $\delta^{13} \mathrm{C}$ & $\delta^{18} \mathrm{O}$ \\
\hline $24 X-2,81$ & 216.01 & 4.5386 & 0.468 & 1.612 \\
\hline $24 X-2,115$ & 216.35 & 4.5469 & 0.647 & 2.460 \\
\hline $24 X-2,141$ & 216.61 & 4.5533 & 0.883 & 2.445 \\
\hline $24 X-3,21$ & 216.91 & 4.5607 & 0.644 & 2.201 \\
\hline $24 X-3,51$ & 217.21 & 4.5680 & 0.999 & 1.770 \\
\hline $24 X-3,81$ & 217.51 & 4.5905 & 0.992 & 2.109 \\
\hline $24 X-3,111$ & 217.81 & 4.6184 & 0.224 & 1.861 \\
\hline $24 X-3,141$ & 218.11 & 4.6463 & 0.392 & 1.648 \\
\hline $24 X-4,21$ & 218.41 & 4.6742 & 0.510 & 1.854 \\
\hline $24 X-4,51$ & 218.71 & 4.7021 & 0.947 & 1.768 \\
\hline $24 X-4,81$ & 219.01 & 4.7300 & 0.842 & 2.071 \\
\hline $24 X-4,111$ & 219.31 & 4.7579 & 0.510 & 1.800 \\
\hline $24 X-4,111$ & 219.31 & 4.7579 & 0.832 & 1.855 \\
\hline $24 X-4,141$ & 219.61 & 4.7822 & 0.775 & 1.754 \\
\hline $24 X-5,21$ & 219.91 & 4.8036 & 0.652 & 1.970 \\
\hline $24 X-5,51$ & 220.21 & 4.8251 & 0.717 & 1.924 \\
\hline $24 X-5,111$ & 220.81 & 4.8680 & 0.779 & 1.870 \\
\hline $24 X-5,141$ & 221.11 & 4.8895 & 1.252 & 1.669 \\
\hline $24 X-6,10$ & 221.30 & 4.9031 & 1.453 & 2.317 \\
\hline $24 X-6,41$ & 221.61 & 4.9253 & 1.048 & 1.985 \\
\hline $24 X-6,71$ & 221.91 & 4.9468 & 0.879 & 1.471 \\
\hline $24 X-6,111$ & 222.31 & 4.9754 & 0.546 & 1.707 \\
\hline $24 X-6,141$ & 222.61 & 4.9969 & 0.943 & 1.946 \\
\hline $24 X-7,41$ & 223.11 & 5.0326 & 1.010 & 1.662 \\
\hline $24 X-7,51$ & 223.21 & 5.0398 & 1.216 & 1.932 \\
\hline $25 X-1,21$ & 223.41 & 5.0541 & 1.734 & 2.269 \\
\hline $25 \mathrm{X}-1,51$ & 223.71 & 5.0756 & 1.464 & 2.463 \\
\hline $25 \times-1,109$ & 224.29 & 5.1171 & 1.088 & 2.053 \\
\hline $25 \mathrm{X}-1,141$ & 224.61 & 5.1400 & 1.177 & 2.013 \\
\hline $25 X-2,21$ & 224.91 & 5.3506 & 1.288 & 1.884 \\
\hline $25 X-2,51$ & 225.21 & 5.3594 & 1.012 & 1.397 \\
\hline $25 \mathrm{X}-2,81$ & 225.51 & 5.3683 & 1.871 & 2.104 \\
\hline $25 X-2,109$ & 225.79 & 5.3765 & 1.334 & 2.120 \\
\hline $25 X-2,141$ & 226.11 & 5.3859 & 0.278 & 1.629 \\
\hline $25 X-2,141$ & 226.11 & 5.3859 & 0.331 & 1.846 \\
\hline $25 X-2,141$ & 227.61 & 5.4301 & 1.139 & 2.086 \\
\hline $25 X-3,21$ & 226.41 & 5.3948 & 0.335 & 1.864 \\
\hline $25 \mathrm{X}-3,21$ & 226.41 & 5.3948 & 0.412 & 2.046 \\
\hline $25 X-3,51$ & 226.71 & 5.4036 & 1.144 & 1.980 \\
\hline $25 \mathrm{X}-3,81$ & 227.01 & 5.4125 & 1.268 & 2.016 \\
\hline $25 X-3,109$ & 227.29 & 5.4207 & 1.095 & 2.012 \\
\hline $25 X-4,21$ & 227.91 & 5.4390 & 0.750 & 2.144 \\
\hline $25 X-4,51$ & 228.21 & 5.4478 & 1.254 & 0.871 \\
\hline $25 \mathrm{X}-4,51$ & 228.21 & 5.4478 & 1.312 & 0.770 \\
\hline $25 X-4,81$ & 228.51 & 5.4567 & 1.288 & 2.504 \\
\hline $25 X-4,109$ & 228.79 & 5.4649 & 1.845 & 2.463 \\
\hline $25 \mathrm{X}-4,141$ & 229.11 & 5.4743 & 1.584 & 2.172 \\
\hline $25 \mathrm{X}-5,21$ & 229.41 & 5.4832 & 1.314 & 2.202 \\
\hline $25 X-5,51$ & 229.71 & 5.4920 & 2.034 & 2.148 \\
\hline $25 X-5,81$ & 230.01 & 5.5008 & 1.758 & 2.634 \\
\hline $25 \mathrm{X}-5,109$ & 230.29 & 5.5091 & 1.026 & 2.404 \\
\hline $25 X-5,141$ & 230.61 & 5.5185 & 1.243 & 1.344 \\
\hline $25 X-6,21$ & 230.91 & 5.5274 & 1.947 & 2.906 \\
\hline $25 \mathrm{X}-6,51$ & 231.21 & 5.5405 & 1.462 & 1.878 \\
\hline $25 X-6,81$ & 231.51 & 5.5555 & 2.109 & 2.627 \\
\hline $25 X-6,109$ & 231.79 & 5.5695 & 1.748 & 2.775 \\
\hline $25 X-6,141$ & 232.11 & 5.5855 & 1.634 & 2.043 \\
\hline $25 X-7,21$ & 232.41 & 5.6005 & 1.200 & 2.438 \\
\hline $25 \times-7,51$ & 232.71 & 5.6155 & 1.828 & 1.964 \\
\hline $26 X-1,51$ & 233.21 & 5.6405 & 1.749 & 1.952 \\
\hline $26 X-1,111$ & 233.81 & 5.6705 & 1.386 & 2.410 \\
\hline $26 X-1,141$ & 234.11 & 5.6831 & 1.285 & 2.101 \\
\hline $26 \mathrm{X}-2,21$ & 234.41 & 5.6915 & 1.488 & 2.447 \\
\hline $26 \mathrm{X}-2,51$ & 234.71 & 5.6999 & 1.253 & 2.354 \\
\hline $26 \mathrm{X}-2,81$ & 235.01 & 5.7083 & 1.351 & 2.330 \\
\hline $26 \mathrm{X}-2,111$ & 235.31 & 5.7167 & 1.603 & 2.411 \\
\hline $26 X-2,141$ & 235.61 & 5.7251 & 1.678 & 2.172 \\
\hline $26 \mathrm{X}-3,21$ & 235.91 & 5.7335 & 1.105 & 2.179 \\
\hline $26 \mathrm{X}-3,51$ & 236.21 & 5.7419 & 1.112 & 1.636 \\
\hline $26 \mathrm{X}-3,111$ & 236.81 & 5.7587 & 1.138 & 2.160 \\
\hline $26 X-3,141$ & 237.11 & 5.7671 & 1.264 & 2.221 \\
\hline $26 X-4,21$ & 237.41 & 5.7755 & 0.970 & 2.191 \\
\hline $26 \mathrm{X}-4,81$ & 238.01 & 5.7923 & 1.271 & 2.149 \\
\hline $26 X-4,81$ & 238.01 & 5.7923 & 1.372 & 2.596 \\
\hline $26 \mathrm{X}-4,111$ & 238.31 & 5.8007 & 1.294 & 2.604 \\
\hline $26 X-5,21$ & 238.91 & 5.8175 & 2.072 & 2.980 \\
\hline $26 \mathrm{X}-5,51$ & 239.21 & 5.8259 & 2.617 & 3.026 \\
\hline
\end{tabular}

Table 3 (continued).

\begin{tabular}{|c|c|c|c|c|}
\hline $\begin{array}{l}\text { Core, section, } \\
\text { interval (cm) }\end{array}$ & $\begin{array}{l}\text { Depth } \\
\text { (mbsf) }\end{array}$ & $\begin{array}{l}\text { Age } \\
\text { (Ma) }\end{array}$ & $\delta^{13} \mathrm{C}$ & $\delta^{18} \mathrm{O}$ \\
\hline $26 \mathrm{X}-5,51$ & 239.21 & 5.8259 & 2.597 & 3.084 \\
\hline $26 \times-5,51$ & 239.21 & 5.8259 & 2.703 & 3.417 \\
\hline $26 \mathrm{X}-6,21$ & 240.41 & 5.8595 & 2.422 & 3.365 \\
\hline $27 \mathrm{X}-1,141$ & & 5.9847 & 1.516 & 1.995 \\
\hline $27 \mathrm{X}-1,141$ & 243.61 & 5.9847 & 1.557 & 1.995 \\
\hline $27 \mathrm{X}-2,51$ & & 6.0116 & 1.205 & 2.371 \\
\hline $27 \mathrm{X}-2,111$ & 244.81 & 6.0385 & 1.380 & 2.023 \\
\hline $27 \mathrm{X}-2,141$ & & 6.0519 & 1.302 & 2.448 \\
\hline $27 \mathrm{X}-2,141$ & 245.11 & 6.0519 & 1.406 & 2.695 \\
\hline $27 \mathrm{X}-3,21$ & 245.41 & 6.0654 & 1.425 & 2.744 \\
\hline $27 \mathrm{X}-3,51$ & 245.71 & 6.0789 & 1.492 & 2.099 \\
\hline $27 X-3,51$ & 245.71 & 6.0789 & 1.618 & 2.425 \\
\hline $27 \mathrm{X}-3,111$ & 246.31 & 6.1058 & 1.515 & 2.471 \\
\hline $27 \mathrm{X}-3,141$ & 246.61 & 6.1192 & 1.420 & 2.074 \\
\hline $27 \mathrm{X}-3,141$ & 246.61 & 6.1192 & 1.445 & 2.038 \\
\hline $27 X-4,15$ & 246.85 & 6.1300 & 1.476 & 2.434 \\
\hline $27 X-4,51$ & 247.21 & 6.1462 & 1.616 & 2.325 \\
\hline $27 \mathrm{X}-4,111$ & 247.81 & 6.1731 & 1.640 & 2.422 \\
\hline $27 \mathrm{X}-4,141$ & 248.11 & 6.1865 & 2.512 & 2.608 \\
\hline $27 \mathrm{X}-4,141$ & 248.11 & 6.1865 & 2.448 & 2.571 \\
\hline $27 \mathrm{X}-4,141$ & 248.11 & 6.1865 & 2.409 & 2.380 \\
\hline $27 \mathrm{X}-4,141$ & 248.11 & 6.1865 & 2.555 & 2.548 \\
\hline $27 X-5,21$ & 248.41 & 6.2000 & 1.766 & 2.628 \\
\hline $27 X-5,51$ & 248.71 & 6.2134 & 1.800 & 2.971 \\
\hline $27 X-5,111$ & 249.31 & 6.2404 & 1.444 & 2.058 \\
\hline $27 \mathrm{X}-6,21$ & 249.91 & 6.2673 & 1.074 & 2.494 \\
\hline $27 X-6,51$ & 250.21 & 6.2807 & 1.581 & 2.556 \\
\hline $27 \mathrm{X}-6,51$ & 250.21 & 6.2807 & 1.593 & 2.779 \\
\hline $27 \mathrm{X}-6,111$ & 25 & 6.3076 & 1.581 & 2.495 \\
\hline $27 \mathrm{X}-6,141$ & 251.11 & 6.3211 & 1.747 & 2.230 \\
\hline $27 X-6,141$ & 251.11 & 6.3211 & 1.670 & 2.359 \\
\hline $27 \mathrm{X}-7,21$ & 251.41 & 6.3346 & 1.850 & 2.503 \\
\hline $27 X-7,51$ & 1 & 6.3 & 1.884 & 2.272 \\
\hline $27 X-7,51$ & 251.71 & 6.3 & 1.826 & 2.437 \\
\hline $28 \mathrm{X}-1,21$ & 251.91 & 6.3570 & 2.286 & 3.087 \\
\hline $28 \mathrm{X}-1,50$ & 252.20 & 6.3700 & 2.271 & 2.877 \\
\hline $28 \mathrm{X}-1,50$ & 2.20 & 6.37 & 2.288 & 2.762 \\
\hline $28 \mathrm{X}-1,100$ & 252.70 & 6.38 & 2.656 & 3.235 \\
\hline 141 & 1 & 6.3 & 2.581 & 3.191 \\
\hline $28 \mathrm{X}-2,21$ & 3.41 & 6.3997 & 2.521 & 3.124 \\
\hline $28 \mathrm{X}-2,50$ & & 6.4 & 2.409 & 2.871 \\
\hline $28 \mathrm{X}-2,80$ & 254.00 & 6.4142 & 2.263 & 2.815 \\
\hline $28 \mathrm{X}-2,80$ & 0 & 6.4 & 2.366 & 2.887 \\
\hline $28 \mathrm{X}-2,100$ & 4.20 & 6.4191 & 2.265 & 3.095 \\
\hline $28 \mathrm{X}-2,141$ & (1) & 6.4 & 1.857 & 2.672 \\
\hline $28 \mathrm{X}-3,21$ & 4.91 & 6.4365 & 1.364 & 2.298 \\
\hline 28 & & 6 & 2.221 & 3.001 \\
\hline $28 \mathrm{X}-3,100$ & 5.70 & 6.4559 & 2.552 & 3.249 \\
\hline (1) & 1 & 6.4 & 2.092 & 2.656 \\
\hline $28 \mathrm{X}-4,21$ & 6.41 & 6.4733 & 2.088 & 2.938 \\
\hline 50 & & & & 2.613 \\
\hline $28 X-4,50$ & 6.70 & 6.4804 & 2.307 & 2.743 \\
\hline 480 & & & 90 & 3.141 \\
\hline $28 \mathrm{X}-4,111$ & 257.31 & 6.4953 & 2.128 & 2.726 \\
\hline 1 & 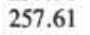 & & & 2.746 \\
\hline $28 \mathrm{X}-5,21$ & 7.91 & 6.5 & 1.643 & 2.477 \\
\hline & & & 2 & 2.978 \\
\hline$-5,50$ & 0 & 6.5 & 2.163 & 3.088 \\
\hline 28 & & & & 2.689 \\
\hline$-5,141$ & 1 & 6.6 & 2.268 & 2.844 \\
\hline & & & & 2.792 \\
\hline$-6,50$ & 0 & 6. & 2.253 & 2.478 \\
\hline & & & & 2.529 \\
\hline 28 & & 6. & 2.230 & 2.898 \\
\hline 2 & & & & 2.702 \\
\hline 141 & & 6.7178 & 2.049 & 2.381 \\
\hline & & & & 2.867 \\
\hline $6-7,50$ & 0 & 6.7272 & 2.329 & 2.695 \\
\hline & & & & 2.801 \\
\hline$-1,51$ & & 6. & 1.767 & 2.535 \\
\hline & & & & 2.378 \\
\hline$-1,141$ & & 6.7498 & 2.292 & 2.914 \\
\hline & & & & \\
\hline $29 \times-2,51$ & & 4 & & 3.037 \\
\hline $29 \times-2,51$ & 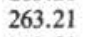 & & & 2.844 \\
\hline$-2,8$ & 263. & 6.7642 & 2.622 & 3.008 \\
\hline $29 \mathrm{X}-2,81$ & 263.51 & 6.7642 & 2.690 & 3.215 \\
\hline
\end{tabular}


Table 3 (continued).

\begin{tabular}{|c|c|c|c|c|}
\hline $\begin{array}{l}\text { Core, section, } \\
\text { interval }(\mathrm{cm})\end{array}$ & $\begin{array}{l}\text { Depth } \\
\text { (mbsf) }\end{array}$ & $\begin{array}{l}\text { Age } \\
\text { (Ma) }\end{array}$ & $\delta^{13} \mathrm{C}$ & $\delta^{18} \mathrm{O}$ \\
\hline $29 X-2,111$ & 263.81 & 6.7690 & 2.406 & 3.105 \\
\hline $29 X-2,141$ & 264.11 & 6.7738 & 2.167 & 2.755 \\
\hline $29 X-3,21$ & 264.41 & 6.7786 & 2.243 & 2.869 \\
\hline $29 \mathrm{X}-3,51$ & 264.71 & 6.7947 & 2.251 & 2.834 \\
\hline $29 X-3,111$ & 265.31 & 6.8367 & 2.214 & 2.792 \\
\hline $29 X-3,141$ & 265.61 & 6.8737 & 2.219 & 2.955 \\
\hline $29 X-4,21$ & 265.91 & 6.9382 & 2.427 & 2.926 \\
\hline $29 X-4,51$ & 266.21 & 7.0027 & 2.068 & 2.568 \\
\hline $29 X-4,81$ & 266.51 & 7.0672 & 1.826 & 2.685 \\
\hline $29 X-4,81$ & 266.51 & 7.0672 & 1.762 & 2.353 \\
\hline $29 X-4,81$ & 266.51 & 7.0672 & 1.802 & 2.370 \\
\hline $29 X-4,110$ & 266.80 & 7.1295 & 1.574 & 2.497 \\
\hline $29 X-4,141$ & 267.11 & 7.1962 & 1.781 & 2.427 \\
\hline $29 X-5,21$ & 267.41 & 7.2607 & 1.607 & 2.654 \\
\hline $29 X-5,51$ & 267.71 & 7.2854 & 2.180 & 2.668 \\
\hline $29 X-5,111$ & 268.31 & 7.3010 & 2.037 & 2.633 \\
\hline $29 X-6,21$ & 268.91 & 7.3166 & 1.954 & 2.752 \\
\hline $29 X-6,51$ & 269.21 & 7.3243 & 1.886 & 1.685 \\
\hline $29 X-6,81$ & 269.51 & 7.3321 & 1.683 & 2.420 \\
\hline $29 X-6,81$ & 269.51 & 7.3321 & 1.676 & 2.287 \\
\hline $29 X-6,81$ & 269.51 & 7.3321 & 1.553 & 1.983 \\
\hline $29 X-6,110$ & 269.80 & 7.3396 & 2.006 & 2.473 \\
\hline $29 X-7,21$ & 270.41 & 7.3525 & 1.831 & 2.630 \\
\hline $29 \times-7,51$ & 270.71 & 7.3561 & 2.308 & 2.369 \\
\hline $29 \times-7,51$ & 270.71 & 7.3561 & 2.288 & 2.498 \\
\hline $29 \times-7,51$ & 270.71 & 7.3561 & 2.120 & 2.220 \\
\hline $29 \times-7,51$ & 270.71 & 7.3561 & 2.196 & 2.069 \\
\hline $30 \times-1,21$ & 270.91 & 7.3584 & 2.630 & 3.345 \\
\hline $30 \times-1,51$ & 271.21 & 7.3621 & 2.563 & 2.999 \\
\hline $30 \times-1,51$ & 271.21 & 7.3621 & 2.634 & 3.193 \\
\hline $30 X-1,111$ & 271.81 & 7.3693 & 1.897 & 2.722 \\
\hline $30 X-1,141$ & 272.11 & 7.3729 & 2.033 & 2.591 \\
\hline $30 X-2,21$ & 272.41 & 7.3765 & 1.947 & 2.841 \\
\hline $30 X-2,37$ & 272.57 & 7.3784 & 1.585 & 2.221 \\
\hline $30 X-2,37$ & 272.57 & 7.3784 & 1.583 & 2.210 \\
\hline $30 \mathrm{X}-2,51$ & 272.71 & 7.3801 & 2.078 & 2.684 \\
\hline $30 X-2,81$ & 273.01 & 7.3837 & 1.444 & 2.190 \\
\hline $30 X-2,111$ & 273.31 & 7.3873 & 1.395 & 2.442 \\
\hline $30 X-3,21$ & 273.91 & 7.3945 & 2.304 & 2.883 \\
\hline $30 X-3,51$ & 274.21 & 7.3981 & 2.450 & 2.728 \\
\hline $30 X-3,111$ & 274.81 & 7.4053 & 2.518 & 3.160 \\
\hline $30 X-3,141$ & 275.11 & 7.4089 & 2.311 & 2.564 \\
\hline $30 X-4,21$ & 275.41 & 7.4170 & 2.271 & 2.864 \\
\hline $30 \times-4,51$ & 275.71 & 7.4269 & 2.233 & 2.606 \\
\hline $30 X-4,81$ & 276.01 & 7.4368 & 2.251 & 2.659 \\
\hline $30 X-4,111$ & 276.31 & 7.4468 & 2.108 & 2.693 \\
\hline $30 X-4,141$ & 276.61 & 7.4567 & 2.347 & 2.942 \\
\hline $30 X-5,21$ & 276.91 & 7.4666 & 2.424 & 2.948 \\
\hline $30 X-5,51$ & 277.21 & 7.4766 & 2.094 & 2.377 \\
\hline $30 X-6,21$ & 278.41 & 7.5163 & 1.887 & 2.749 \\
\hline $30 X-7,20$ & 279.90 & 7.5656 & 1.683 & 2.630 \\
\hline $30 \times-7,51$ & 280.21 & 7.5759 & 1.528 & 2.274 \\
\hline $30 \times-7,51$ & 280.21 & 7.5759 & 1.570 & 2.296 \\
\hline $31 X-1,21$ & 280.41 & 7.5825 & 1.960 & 2.448 \\
\hline $31 \times-1,51$ & 280.71 & 7.5924 & 1.957 & 2.377 \\
\hline $31 X-1,111$ & 281.31 & 7.6123 & 1.768 & 2.685 \\
\hline $31 X-1,141$ & 281.61 & 7.6222 & 1.518 & 2.189 \\
\hline $31 X-2,20$ & 281.90 & 7.6318 & 1.355 & 2.046 \\
\hline $31 \mathrm{X}-2,51$ & 282.21 & 7.6421 & 1.472 & 1.993 \\
\hline $31 X-2,81$ & 282.51 & 7.6520 & 1.915 & 2.543 \\
\hline $31 X-2,111$ & 282.81 & 7.6620 & 1.872 & 2.779 \\
\hline $31 X-2,141$ & 283.11 & 7.6719 & 2.169 & 2.705 \\
\hline $31 X-3,20$ & 283.40 & 7.6815 & 2.198 & 2.986 \\
\hline $31 X-3,51$ & 283.71 & 7.6918 & 2.476 & 2.909 \\
\hline $31 X-3,111$ & 284.31 & 7.7116 & 2.530 & 3.241 \\
\hline $31 X-3,141$ & 284.61 & 7.7216 & 2.275 & 2.611 \\
\hline $31 X-4,20$ & 284.90 & 7.7312 & 2.234 & 2.672 \\
\hline $31 X-4,81$ & 285.51 & 7.7513 & 2.191 & 2.441 \\
\hline $31 X-4,141$ & 286.11 & 7.7712 & 1.745 & 2.055 \\
\hline $31 X-5,20$ & 286.40 & 7.7808 & 1.788 & 2.457 \\
\hline $31 X-5,51$ & 286.71 & 7.7911 & 1.759 & 2.421 \\
\hline $31 X-5,111$ & 287.31 & 7.8106 & 1.936 & 2.336 \\
\hline $31 X-5,141$ & 287.61 & 7.8209 & 2.243 & 2.498 \\
\hline $31 X-6,20$ & 287.90 & 7.8305 & 1.539 & 2.189 \\
\hline $31 X-6,51$ & 288.21 & 7.8407 & 2.007 & 2.272 \\
\hline $31 X-6,81$ & 288.51 & 7.8507 & 1.362 & 2.008 \\
\hline $31 X-6,110$ & 288.80 & 7.8603 & 1.386 & 2.050 \\
\hline $31 X-6,141$ & 289.11 & 7.8705 & 1.970 & 2.057 \\
\hline $31 X-7,46$ & 289.66 & 7.8887 & 2.348 & 2.886 \\
\hline
\end{tabular}

$3.5 \%$ oo. At this point, $\delta^{18} \mathrm{O}$ decreases rapidly to $2.5 \%$ o by 170 mbsf $(2.67 \mathrm{Ma})$, where it oscillates about the mean with variability of $0.3 \%$ to $5 \%$ to the present day. Variability increases above about $40 \mathrm{mbsf}$. A temporary maximum occurs at 11 to 5 mbsf $(0.49-0.31 \mathrm{Ma})$, followed by a local minimum at about $2.5 \mathrm{mbsf}(0.24 \mathrm{Ma})$.

\section{Carbon Isotopes}

The record of fine-fraction $\delta^{13} \mathrm{C}$ shows a different character than that of $\delta^{18} \mathrm{O}$ (Fig. 2 and Table 3). Between 290 and about $255 \mathrm{mbsf}(7.9-6.44 \mathrm{Ma}), \delta^{13} \mathrm{C}$ is roughly constant, averaging about $2 \%$. At this point, fine-fraction $\delta^{13} \mathrm{C}$ begins to decrease, reaching a minimum of $0.33 \%$ at 210.2 mbsf $(4.23$ Ma). This decrease in $\delta^{13} \mathrm{C}$, roughly coincident with the late Miocene carbon shift (Keigwin and Shackleton, 1980), is observed in the planktonic and benthic foraminiferal records, but the shift in the fine fraction occurs across a period of about 2 m.y. rather than a few hundred thousand years as found in the foraminiferal records. The sampling frequency is somewhat less from this point upward, but it appears that $\delta^{13} \mathrm{C}$ becomes more variable, with spikes of high $\delta^{13} \mathrm{C}$ values occurring at about $203.5 \mathrm{mbsf}$ ( $4.06 \mathrm{Ma}$ ), $181 \mathrm{mbsf}$ (3.17 Ma), $175 \mathrm{mbsf}(2.86 \mathrm{Ma})$, and $171 \mathrm{mbsf}(2.71 \mathrm{Ma})$.

Between 170.41 and $168.91 \mathrm{mbsf}(2.69-2.55 \mathrm{Ma})$, a sudden shift in $\delta^{13} \mathrm{C}$ occurs, with $\delta^{13} \mathrm{C}$ falling from an average of $1.26 \%$ oo between the base of the Gauss and the shift to an average of $-0.22 \%$ between the shift and the top of the Matuyama, for a total change of $-1.48 \%$.

The amplitude of variability above the Gauss/Matuyama boundary is small up to about $75 \mathrm{mbsf}(1.46 \mathrm{Ma})$. Above 75 mbsf, large swings in $\delta^{13} \mathrm{C}$ occur, with both the highest and lowest $\delta^{13} \mathrm{C}$ values measured at Site 704 found in this interval. Maxima occur at $61.00 \mathrm{mbsf}(1.24 \mathrm{Ma}), 36.51 \mathrm{mbsf}(0.78 \mathrm{Ma})$, and $5.75 \mathrm{mbsf}(0.33 \mathrm{Ma})$. The increase to the $36.51 \mathrm{mbsf}$ maximum is fairly smooth, beginning with a minimum of $1.84 \%$ at $52.90 \mathrm{mbsf}(1.11 \mathrm{Ma})$ and ending at $3.23 \%$ oo. A similar trend occurs in the buildup to a 6 mbsf maximum, starting with a minimum of $-2.30 \%$ at $17.91 \mathrm{mbsf}(0.63 \mathrm{Ma})$ and increasing to $3.14 \%$ oo.

The meaning of the younger events is unclear, because of the disturbed nature of the upper $50 \mathrm{~m}$ (Shipboard Scientific Party, 1988). This disturbance can affect our knowledge of the timing and magnitude of the events. However, core disturbance would be expected to homogenize and decrease the magnitude of the signals seen. The magnitude of these changes (up to $5.52 \%$ oo for $\delta^{13} \mathrm{C}$ between 36.51 and $28.66 \mathrm{mbsf}$ and $2.77 \%$ oo for $\delta^{18} \mathrm{O}$ between 32.91 and $28.66 \mathrm{mbs}$ ) must therefore be minimum magnitudes. Clearly, extremely large changes in fine-fraction isotopic composition in the upper $75 \mathrm{~m}$ of Site 704 have occurred.

\section{Changes in the Fine-Fraction Composition}

Results of random-settling slide analysis show that changes have indeed occurred in the particle composition of the fine fraction (Fig. 4 and Table 4). Significant changes occurred at $170-160,150-140,110-100$, and $90-80$ mbsf and every $10 \mathrm{~m}$ above the latter depth to a depth of $60 \mathrm{mbsf}$, the shallowest sample thus far examined. From 170 to $160 \mathrm{mbsf}$, the nannofossil content of the fine fraction drops from over $90 \%$ to about $40 \%$, whereas micrite content rises from near $0 \%$ to approximately $60 \%$. From 150 to $140 \mathrm{mbsf}$, nannofossil content rises again to about $90 \%$, whereas micrite content falls to about $10 \%$. Nannofossil content again drops to about $50 \%$ between 110 and 100 mbsf with micrite rising proportionately. Nannofossil and micrite contents fluctuate widely every $10 \mathrm{~m}$ above this point, with foraminiferal content becoming important only at $60 \mathrm{mbsf}$. There is a general increase upward of the micrite content, contrary to the 

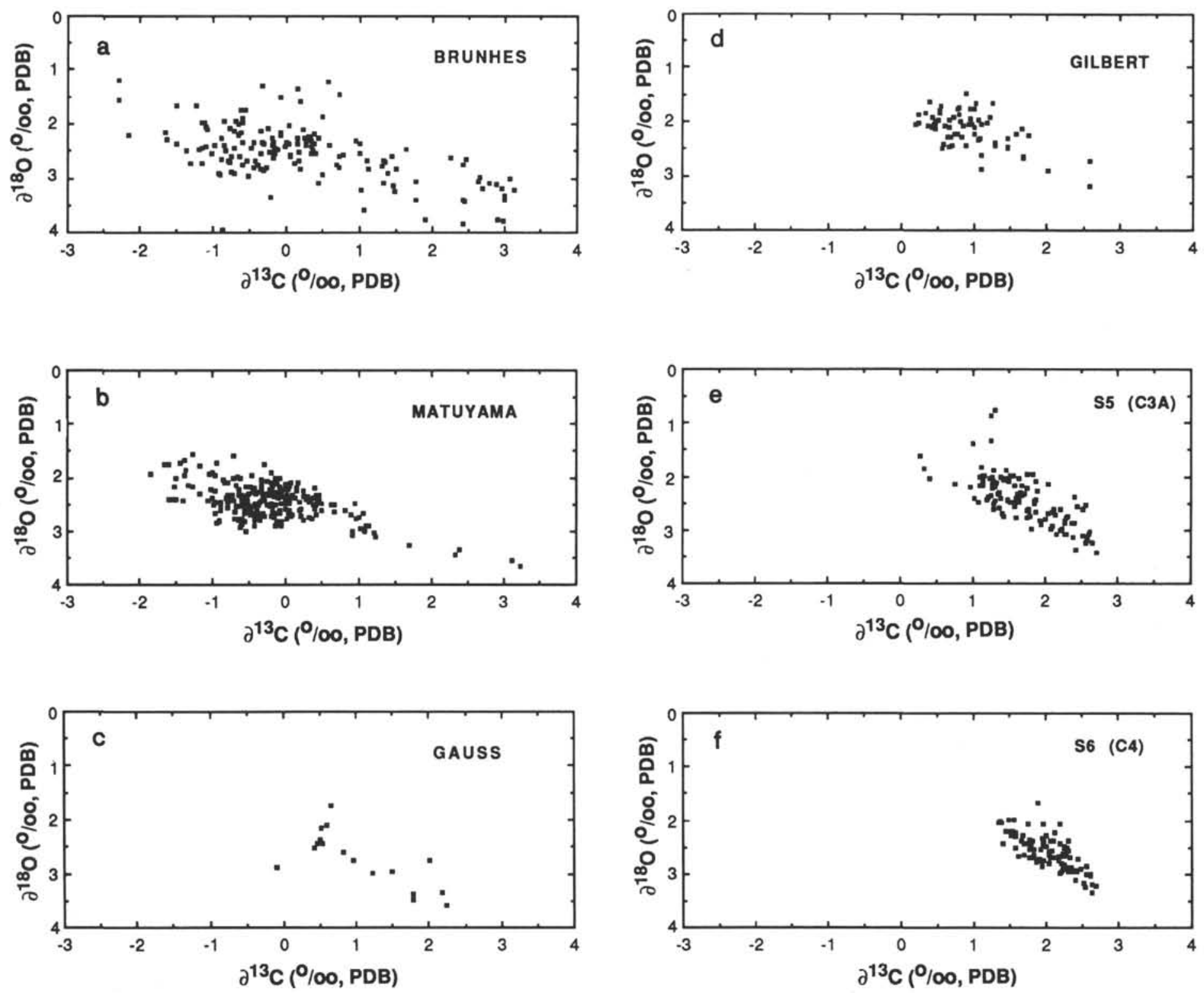

Figure 3. Covariance between fine-fraction $\delta^{13} \mathrm{C}$ and $\delta^{18} \mathrm{O}$ for different paleomagnetic polarity intervals: Brunhes $(0-34.51 \mathrm{mbsf}, 0-0.73 \mathrm{Ma})$, Matuyama (34.51-168.72 mbsf, 0.73-2.47 Ma), Gauss (168.72-189.69 mbsf, 2.47-3.40 Ma), Gilbert (189.69-224.89 mbsf, 3.40-5.35 Ma), S5 (C3A) (224.89-259.50 mbsf, 5.35-6.70 Ma), and S6 (C4) (259.50-290.00 mbsf, 6.70-7.90 Ma). 


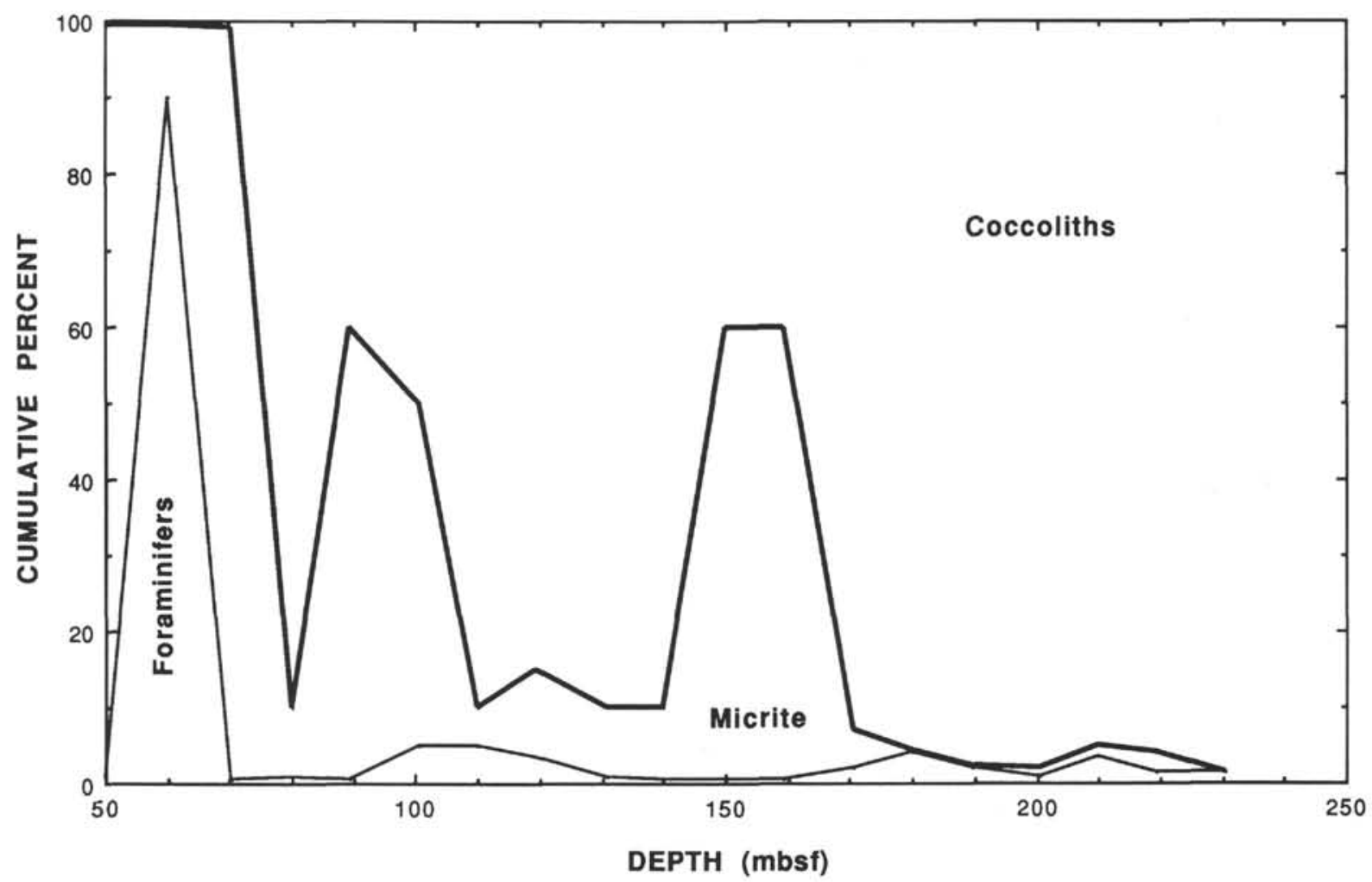

Figure 4. Random-settling slide analysis vs. depth.

downward increase that might be expected if diagenesis increased downhole.

\section{DISCUSSION}

This discussion will deal primarily with the large $\delta^{13} \mathrm{C}$ shift at $2.6 \mathrm{Ma}$; it will also touch on some aspects of the large

Table 4. Estimated fine-fraction composition from randomsettling slide analysis, Hole 704A.

\begin{tabular}{|c|c|c|c|c|}
\hline $\begin{array}{l}\text { Core, section, } \\
\text { interval }(\mathrm{cm})\end{array}$ & $\begin{array}{l}\text { Depth } \\
\text { (mbsf) }\end{array}$ & $\begin{array}{c}\text { Foraminifers } \\
(\%)\end{array}$ & $\begin{array}{c}\text { Calcareous } \\
\text { nannofossils } \\
(\%)\end{array}$ & $\begin{array}{c}\text { Micrite } \\
(\%)\end{array}$ \\
\hline $6 \mathrm{H}-4,20$ & 49.90 & 0.5 & 0.5 & 99 \\
\hline $7 \mathrm{H}-4,91$ & 60.11 & 89.5 & 0.5 & 10 \\
\hline $7 \mathrm{H}-4,91$ & 60.11 & 89.5 & 0.5 & 10 \\
\hline $8 \mathrm{H}-4,140$ & 70.10 & 0.5 & 1 & 98.5 \\
\hline $9 \mathrm{H}-5,21$ & 79.91 & 1 & 90 & 9 \\
\hline $10 \mathrm{H}-5,20$ & 89.40 & 0.5 & 40 & 59.5 \\
\hline $10 \mathrm{H}-5,20$ & 89.40 & 0.5 & 40 & 59.5 \\
\hline $11 \mathrm{H}-6,21$ & 100.41 & 5 & 50 & 45 \\
\hline $11 \mathrm{H}-6,21$ & 100.41 & 5 & 50 & 45 \\
\hline $12 \mathrm{H}-6,21$ & 109.91 & 5 & 90 & 5 \\
\hline $13 \mathrm{H}-6,21$ & 119.41 & 4 & 86 & 10 \\
\hline $15 \mathrm{H}-1,21$ & 130.91 & 1 & 90 & 9 \\
\hline $15 \mathrm{H}-1,21$ & 130.91 & 1 & 90 & 9 \\
\hline $15 \mathrm{H}-1,21$ & 130.91 & 1 & 90 & 9 \\
\hline $15 \mathrm{H}-7,21$ & 139.91 & 0.5 & 89.5 & 10 \\
\hline $17 X-1,21$ & 149.91 & 0.5 & 40 & 59.5 \\
\hline $18 \mathrm{X}-1,21$ & 159.41 & 0.5 & 40 & 59.5 \\
\hline $19 X-2,21$ & 170.41 & 2 & 93 & 5 \\
\hline $20 \mathrm{X}-2,21$ & 179.91 & 4 & 95.5 & 0.5 \\
\hline $20 \mathrm{X}-2,21$ & 179.91 & 4 & 95.5 & 0.5 \\
\hline $21 X-2,46$ & 189.66 & 2 & 97.5 & 0.5 \\
\hline $21 X-2,46$ & 189.66 & 2 & 97.5 & 0.5 \\
\hline $22 \mathrm{X}-3,21$ & 200.41 & 3.5 & 95 & 1.5 \\
\hline $23 \mathrm{X}-3,21$ & 209.91 & 3.5 & 95 & 1.5 \\
\hline $24 \mathrm{X}-3,21$ & 219.41 & 1.5 & 94 & 4.5 \\
\hline $25 X-4,21$ & 230.41 & 1.5 & 98.5 & 0 \\
\hline
\end{tabular}

changes in $\delta^{13} \mathrm{C}$ and $\delta^{18} \mathrm{O}$ above $50 \mathrm{mbsf}$ in the fine-fraction record.

Several possible causes for changes in the Site 704 finefraction isotope record exist:

1. diagenetic effects;

2. changes in the fine-fraction composition

a. change in the nannofossil assemblage

b. change in percent of micrite or of juvenile or broken planktonic or benthic foraminifers;

3. paleoceanographic change

a. changes in upwelling and primary productivity of surface waters

b. movement of the Antarctic Polar Front.

\section{Diagenetic Effects}

Initial examination of the preservation of the fine fraction found that slight etching of nannofossils occurs above Core 114-704B-10H (82.7-92.2 mbsf), and progressive overgrowth by secondary calcite below (Shipboard Scientific Party, 1988). We wish to determine whether this etching and overgrowth may have affected fine-fraction isotopic composition.

One indicator of a diagenetic overprint on isotopic composition would be for the isotopic composition of the fine fraction and/or planktonic foraminifers to follow that of the benthic foraminifers. Comparison of $\delta^{18} \mathrm{O}$ records deeper than about $223 \mathrm{mbsf}(5.0 \mathrm{Ma})$ (Fig. 5) shows that $\delta^{18} \mathrm{O}$ of the fine fraction and of the benthic foraminifers are nearly the same, but that $\delta^{18} \mathrm{O}$ of Neogloboquadrina pachyderma averages $0.5 \%$ oo to $1.0 \%$ oo lighter. Inspection of the carbon isotopic records (Fig. 6) reveals that fine-fraction and benthic foraminiferal $\delta^{13} \mathrm{C}$ average about the same value in the interval from 170 to $58.5 \mathrm{mbsf}(2.7-1.2 \mathrm{Ma})$, although $\delta^{18} \mathrm{O}$ seems uncorrelated. 
Diagenesis commonly affects $\delta^{18} \mathrm{O}$ through exchange with pore waters or bottom waters. However, it rarely affects $\delta^{13} \mathrm{C}$ in Cenozoic sediments because relatively little carbon is present in bottom waters or pore waters compared to that contained in carbonate on the seafloor or in sediments (Garrison, 1981). Therefore, we consider it unlikely that finefraction carbonate $\delta^{13} \mathrm{C}$ would have been affected by diagenesis in the 170-58.5 mbsf interval without also affecting $\delta^{18} \mathrm{O}$. In the 223-289.66 mbsf interval we suggest that although $\delta^{18} \mathrm{O}$ values of the fine fraction may have been affected by diagenesis, $\delta^{13} \mathrm{C}$ values have not.

\section{Effects of Fine-Fraction Composition}

Because a large shift in $\delta^{13} \mathrm{C}$ occurs at $170-160 \mathrm{mbsf}$ (Fig. 6 ), it is conceivable that the shift was caused by the change in fine-fraction composition at this point. This may well have had an effect, but it should also be noted that when the finefraction returns to close to the previous bulk composition in the $150-140$ mbsf interval, the $\delta^{13} \mathrm{C}$ values do not return to the values from below $170 \mathrm{mbsf}$ nor do the compositional changes correlate to significant isotopic changes (Fig. 7). This argues against fine-fraction composition alone controlling isotopic composition. Because of the paucity of analyses in the Gauss, immediately below 170 mbsf, it is difficult to say how the covariance between $\delta^{13} \mathrm{C}$ and $\delta^{18} \mathrm{O}$ changes, but the shift in $\delta^{13} \mathrm{C}$ is easily seen in examination of records from sediments above and below the Gauss/Matuyama boundary.

\section{Paleoceanographic Change}

The final possible cause of the shift in carbon isotopic values is a change in paleoceanographic conditions. Two interpretations of the decrease in $\delta^{13} \mathrm{C}$ of fine-fraction carbonate along these lines are possible:

1. Decreased primary productivity leading to enrichment in ${ }^{12} \mathrm{C}$ in surface waters.

2. A northward shift in the position of the Antarctic Polar Front, causing an increase in upwelling rates.

Decreased primary productivity would allow ${ }^{12} \mathrm{C}$ to accumulate in surface waters, decreasing $\delta^{13} \mathrm{C}$ instead of being preferentially extracted by plants. However, a decrease in primary productivity is not really a viable interpretation. Although carbonate content falls from an average of $72.7 \%$ to $42.7 \%$, sedimentation rates increase significantly above the Gauss/Matuyama boundary, from an average of 22.3 $\mathrm{m} / \mathrm{m}$.y. to 3.3 times that, $73.0 \mathrm{~m} / \mathrm{m}$.y. Assuming that sediment densities remain constant, this would imply that accumulation rates of carbonate after the Gauss/Matuyama boundary increased by $3.3 \times(42.7 / 72.7)=1.94$ times the pre-boundary value. Inspection of the sediments shows instead that lithologies change to become more biosiliceousrich slightly below the boundary, at $178 \mathrm{mbsf}(2.99 \mathrm{Ma})$ (Shipboard Scientific Party, 1988). This implies increased biosiliceous productivity as well. The increased sedimentation rate thus appears to be due to an increase in productivity of overlying waters, of both siliceous and calcareous components (Hodell and Ciesielski, 1990).

If a paleoceanographic change did lead to a change in $\delta^{13} \mathrm{C}$ of total $\mathrm{CO}_{2}$, that change probably was a northward shift in the position of the Polar Front. This would explain several features seen in the Site 704 sediments. Increased upwelling at the site would lead to increased productivity and therefore to an increased sedimentation rate. Second, the $\delta^{13} \mathrm{C}$ of surface water would decrease. Third, as productivity increased, more organic carbon would be deposited on the ocean floor, which might help explain the simultaneous decrease seen in $\delta^{13} \mathrm{C}$ of benthic foraminifers (but see Hodell and Ciesielski, this vol- ume, whose alternate interpretation suggests a change in deep-water $\delta^{13} \mathrm{C}$, a more likely explanation).

Several investigations have identified a trend of northward movement of the Polar Front from at least 2.9 to $1.9 \mathrm{Ma}$. Ciesielski and Weaver (1983) documented a northward shift in the position of the Polar Front over DSDP Site 514 (latitude $46^{\circ} 03^{\prime} \mathrm{S}$ ) on the Falkland Plateau at 2.7-2.6 Ma, after which oscillations across the site occurred to at least $0.7 \mathrm{Ma}$. They based their findings on changes in radiolarian assemblages. Ciesielski and Grinstead (1986) refined the study of Site 514 by applying factor analysis to the radiolarian assemblages. Their results indicate increasing importance of Antarctic forms from 2.94 to $2.68 \mathrm{Ma}$, suggesting a progressive northward movement of the APFZ. From 2.67 to $1.91 \mathrm{Ma}$ the Polar Front remained north of Site 514. Westall and Fenner (this volume) interpreted diatom and lithostratigraphic data from Hole 704B to infer northward movement of the APFZ at $101.7 \mathrm{mbsf}(0.94$ or $1.78 \mathrm{Ma}$ according to their age models, $1.94 \mathrm{Ma}$ according to our model-see Hodell and Ciesielski, this volume, for discussion of age models). At this depth, they found a further increase in biosiliceous content and a decrease in calcareous content. We would suggest that our data document movement of the northern boundary of the APFZ over Site 704 at 2.47 $\mathrm{Ma}$, increasing carbonate and bulk accumulation rates, and that the data of Westall and Fenner (this volume) document the movement of the southern edge over Site 704 at $1.94 \mathrm{Ma}$, decreasing carbonate content but keeping total sedimentation rates high.

An increase in upwelling or mixing does not address the question of why the Neogloboquadrina pachyderma record does not show a similar depletion in $\delta^{13} \mathrm{C}$ and why there is a consequent change in the fine fraction $/ N$. pachyderma $\delta^{13} \mathrm{C}$ gradient. The gradient between $\delta^{13} \mathrm{C}$ of $N$. pachyderma and the fine fraction changes as a result of the shift in $\delta^{13} \mathrm{C}$. The $\delta^{13} \mathrm{C}$ of $N$. pachyderma averages $0.58 \%$ from the base of the Gauss to the shift, and virtually the same $(0.59 \%)$ from the shift to the top of the Matuyama, whereas the average $\delta^{13} \mathrm{C}$ of the fine fraction changes from $1.26 \%$ to $-0.22 \%$, respectively, in these same intervals. This represents a change in the fine fraction $/ N$. pachyderma $\delta^{13} \mathrm{C}$ gradient from $0.68 \%$ to $-0.81 \%$ o, a difference of $1.49 \%$ oo.

$N$. pachyderma has been characterized as a deep-living species, depositing much of its calcite under near-isothermal conditions or below the thermocline (Srinivasan and Kennett, 1974; Reynolds and Thunell, 1986). This suggests that the fine fraction is recording $\delta^{13} \mathrm{C}$ of shallower water, while $N$. pachyderma is recording $\delta^{13} \mathrm{C}$ of deeper, less variable water masses. Fine-fraction $\delta^{13} \mathrm{C}$ would then be affected by the rate of upwelling and thus the position of the APFZ, which would control the balance between productivity and upwelling rate. $N$. pachyderma $\delta^{13} \mathrm{C}$ would only record the average $\delta^{13} \mathrm{C}$ of the upwelling water, which may not be affected by the rate of upwelling.

Because both the fine fraction (Margolis et al., 1975; Goodney et al., 1980; Paull and Theirstein, 1987) and N. pachyderma (Williams et al., 1977; Kahn and Williams, 1981; Charles and Fairbanks, 1990) deposit their calcite out of equilibrium with seawater- $\Sigma \mathrm{CO}_{2}$, it is difficult to interpret the meaning of this gradient change. Both groups produce lower $\delta^{13} \mathrm{C}$ values than equilibrium. In the subantarctic, $N$. pachyderma is approximately $1.0 \%$ oo too low (Charles and Fairbanks, 1990); therefore, we can use this value as a correction factor. However, the fine fraction produces $\delta^{13} \mathrm{C}$ with variable relationships to equilibrium calcite; it is always light, but the correction varies.

Whether an increase in the upwelling to primary productivity ratio caused by a shift in the position of the APFZ is 
responsible for the change in $\delta^{13} \mathrm{C}$ at $2.6 \mathrm{Ma}$, or whether it is due to compositional change in the fine fraction, is somewhat problematical. Assemblage changes did occur at this time; this, coupled with the lack of an equivalent change in $\delta^{13} \mathrm{C}$ in $N$. pachyderma, supports the interpretation that a change in the fine-fraction composition is at least partly responsible for isotopic changes. On the other hand, changes in gross composition that are not reflected in $\delta^{13} \mathrm{C}$ occurred at other times as well (see Fig. 4). In addition, the very fact that the fine-fraction composition does change slightly before the Gauss/Matuyama boundary is evidence for the existence of environmental control.

Within the Matuyama, from $168.72 \mathrm{mbsf}(2.47 \mathrm{Ma})$ to about $90 \mathrm{mbsf}(1.7 \mathrm{Ma})$, the $\delta^{13} \mathrm{C}$ of the fine fraction and of $N$. pachyderma exhibit a rough covariance. This suggests that after the APFZ moved north of Site 704, upwelling was intense enough for the $\delta^{13} \mathrm{C}$ of the upwelled water to control the $\delta^{13} \mathrm{C}$ of both the fine fraction and $N$. pachyderma.

The very large amplitude variations in $\delta^{13} \mathrm{C}$ and $\delta^{18} \mathrm{O}$ in the upper $70 \mathrm{~m}$ (mostly Brunhes paleomagnetic Epoch) of Site 704 deserve some mention. However, their interpretation is made difficult by the fact that because of the disturbance in this section, our knowledge of the chronostratigraphy is poor. This section exhibits some of the most extreme covariance between $\delta^{13} \mathrm{C}$ and $\delta^{18} \mathrm{O}$ at the site (Fig. 3). Goodney et al. (1980) demonstrated that the fine fraction shows a high degree of covariance. Paull and Theirstein (1987) demonstrated that this covariance is not necessarily related to water properties but instead to assemblage changes and that the assemblage changes can also lead to large changes in isotopic composition. Large changes in fine-fraction composition begin at about $80 \mathrm{mbsf}$ and continue to at least $50 \mathrm{mbsf}$ (Fig. 4), and we feel that the most likely source for the large isotopic variations is these compositional changes. To investigate this possibility further, changes both in gross composition (foraminifers vs. calcareous nannofossils vs. micrite percentages) and in species composition need to be addressed in the future. Although we do not yet understand the complete meaning of these isotopic changes, it should be noted that the changes in fine-fraction composition that occur simultaneously with these isotopic changes suggest variations in paleoceanographic conditions that also should be investigated.

\section{CONCLUSIONS}

Isotopic analyses of the fine-fraction carbonate of ODP Site 704 have supplemented isotopic analyses of Neogloboquadrina pachyderma and benthic foraminifers. The fine-fraction analyses revealed a shift toward lighter $\delta^{13} \mathrm{C}$ at $2.6 \mathrm{Ma}$, corresponding to (1) a large increase in the sedimentation rate in Site 704 and (2) a simultaneous, almost identical shift in $\delta^{13} \mathrm{C}$ in the benthic foraminiferal record. The fine fraction is made up of many components, and it is difficult to determine the exact cause of this shift. We suggest that it may be due to one or a combination of the following causes:

1. Changes in the fine-fraction composition.

a. Change in the nannofossil assemblage.

b. Change in percent of juvenile or broken planktonic or benthic foraminifers.

2. Increased upwelling of ${ }^{12} \mathrm{C}$-enriched deep water, caused by a shift in the position of the Antarctic Polar Front.

This shift is part of a progressive northward movement of the APFZ, begun at least by $2.94 \mathrm{Ma}$ (Ciesielski and Grinstead, 1986) and lasting at least until 1.90 Ma (Westall and Fenner, this volume).

The shift in $\delta^{13} \mathrm{C}$ at this time is not seen in the $\delta^{13} \mathrm{C}$ record from $N$. pachyderma, for reasons that are not clear at this time. We suggest that the reason for this may be the deposition of fine-fraction calcite primarily in depleted surface water, which is more affected by changes in upwelling rates than at deeper water levels, where the foraminifer $N$. pachyderma secretes its test.

\section{ACKNOWLEDGMENTS}

We thank two anonymous reviewers for making substantial improvements to the manuscript and to our conclusions. One of the reviewers suggested the deep-living habitat of $N$. pachyderma as the reason for the lack of correlation between carbon isotope records of foraminiferal and fine-fraction carbonate. GAM, DAH, and DWM did the isotopic analyses and thank R. Cooke for sample preparation and general laboratory assistance. PFC produced the stratigraphic framework and assisted in the fine-fraction compositional analyses.

This work was supported by a University of Florida Graduate Council Fellowship to GAM, U.S. Science Program (USSAC) Grant 20114 and NSF Grant OCE-8858012 to DAH, USSAC Grant 20115 to PFC, and NSF Grant DPP-8717854 to both DAH and PFC.

\section{REFERENCES}

Anderson, T. F., and Cole, S. A., 1975. The stable isotope geochemistry of marine coccoliths: a preliminary comparison with planktonic Foraminifera. J. Foraminiferal Res., 5:188-192.

Barrera, E., and Savin, S. M., 1987. Effect of sample preparation on the $\delta^{18} \mathrm{O}$-value of fine-grained calcite. Chem. Geol., 66:301-305.

Berggren, W. A., Kent, D. V., Flynn, J. J., and Van Couvering, J. A., 1985. Cenozoic geochronology. Geol. Soc. Am. Bull., 96:1499-1510.

Charles, C. D., and Fairbanks, R. G., 1990. Glacial to interglacial changes in the isotopic gradients of Southern Ocean surface water. In Bleil, U., and Thiede, J. (Eds.), Geologic History of the Polar Oceans: Arctic versus Antarctic: NATO ASI Ser., 519-538.

Ciesielski, P. F., and Grinstead, G. P., 1986. Pliocene variations in the position of the Antarctic Convergence in the southwest Atlantic. Paleoceanography, 1:197-232.

Ciesielski, P. F., and Weaver, F. M., 1983. Neogene and Quaternary paleoenvironmental history of Deep Sea Drilling Project Leg 71 sediments, southwest Atlantic Ocean. In Ludwig, W. J., Krasheninnikov, V. A., et al., Init. Repts. DSDP, 71: Washington (U.S. Govt. Printing Office), 461-477.

Dudley, W. C., Blackwelder, P. L., Brand, L. E., and Duplessy, J. C., 1986. Stable isotopic composition of coccoliths. Mar. Micropaleontol., 10:1-8.

Dudley, W. C., Duplessy, J. C., Blackwelder, P. L., Brand, L. E., and Guillard, R.R.L., 1980. Coccoliths in Pleistocene-Holocene nannofossil assemblages. Nature, 285:222-223.

Garrison, R., 1981. Diagenesis of ocean carbonate sediments: a review of the DSDP perspective. In Warme, J. E., Douglas, R. G., and Winterer, E. L. (Eds.), The Deep Sea Drilling Project: A Decade of Progress: Soc. Econ. Paleontol. Mineral. Spec. Publ., 32:181-207.

Goodney, D. E., Margolis, S. V., Dudley, W. C., Kroopnick, P., and Williams, D. F., 1980. Oxygen and carbon isotopes of Recent calcareous nannofossils as paleoceanographic indicators. Mar. Micropaleont., 5:31-42.

Hailwood, E., 1989. The role of magnetostratigraphy in the development of geological time scales. Paleoceanography, 4:1-18.

Hodell, D. A., Benson, R. H., Kennett, J. P., and Rakic, K.E.B., 1989. Stable isotope stratigraphy of late Miocene-early Pliocene sequences in northwest Morocco: the Bou Regreg Section. Paleoceanography, 4:467-482

Hodell, D. A., and Ciesielski, P. F., 1990. Southern Ocean response to the onset of Northern Hemisphere glaciation at 2.4 Ma. In Bleil, U., and Thiede, J. (Eds.), Geologic History of the Polar Oceans: Arctic versus Antarctic: NATO ASI Ser., 707-728.

Kahn, M. I., and Williams, D. F., 1981. Oxygen and carbon isotopic composition of living planktonic foraminifers from the northeast Pacific Ocean. Palaeogeogr., Palaeoclimatol., Palaeoecol., $33: 47-69$. 
Keigwin, L. D., Jr., and Shackleton, N. J., 1980. Uppermost Miocene carbon isotope stratigraphy of a piston core in the equatorial Pacific. Nature, 284:613-614.

Knox, F., and McElroy, M. B., 1984. Changes in atmospheric $\mathrm{CO}_{2}$ : influence of the marine biota at high latitude. J. Geophys. Res., 89:4629-4637.

Kroopnick, P., 1980. The distribution of ${ }^{13} \mathrm{C}$ in the Atlantic Ocean. Earth Planet. Sci. Lett., 49:469-484.

1985. The distribution of ${ }^{13} \mathrm{C}$ of $\mathrm{\Sigma CO}_{2}$ in the world oceans. Deep-Sea Res., Part A, 32:57-84.

Lutjeharms, J.R.E., 1985. Location of frontal systems between Africa and Antarctica: some preliminary results. Deep-Sea Res., Part A, 32:1499-1509.

Margolis, S. V., Kroopnick, P. M., Goodney, D. E., Dudley, W. C., and Mahoney, M. E., 1975. Oxygen and carbon isotopes from calcareous nannofossils as paleoceanographic indicators. Science, 189:555-557.

Matthews, R. K., and Poore, R. Z., 1980. Tertiary $\delta^{18} \mathrm{O}$ record and glacio-eustatic sea-level fluctuations. Geology, 8:501-504.

Miller, K. G., and Fairbanks, R. G., 1983. Evidence for Oligocenemiddle Miocene abyssal circulation changes in the western North Atlantic. Nature, 306:250-253.

Miller, K. G., Mountain, G. S., and Tucholke, B. E., 1985. Oligocene glacio-eustasy and erosion on the margins of the North Atlantic. Geology, 13:10-13.

Moore, T. C., 1973. Method of randomly distributing grains for microscopic examination. J. Sediment. Petrol., 43:904-906.

Paull, C. K., and Thierstein, H. R., 1987. Stable isotopic fractionation among particles in quaternary coccolith-sized deep-sea sediments. Paleoceanography, 2:423-429.

Raymo, M. E., Ruddiman, W. F., Backman, J., Clement, B. M., and Martinson, D. G., 1989. Late Pliocene variation in Northern Hemisphere ice sheets and North Atlantic deep water circulation. Paleoceanography, 4:413-446.
Reynolds, L. A., and Thunell, R. C., 1986. Seasonal production and morphologic variation of Neogloboquadrina pachyderma (Ehrenberg) in the northeast Pacific. Micropaleontology, 32:1-18.

Ruddiman, W. F., Raymo, M., and McIntyre, A., 1986. Matuyama 41,000-year cycles: North Atlantic Ocean and Northern Hemisphere ice sheets. Earth Planet. Sci. Lett., 80:117-129.

Ruddiman, W. F., Raymo, M. E., Martinson, D. G., Clement, B. M., and Backman, J., 1989. Pleistocene evolution: Northern Hemisphere ice sheets and North Atlantic Ocean. Paleoceanography, 4:353-412.

Shackleton, N. J., Backman, J., Zimmerman, H., Kent, D. V., Hall, M. A., Roberts, D. G., Schnitker, D., Baldauf, J., Desprairies, A., Homrighausen, R., Huddlestun, P., Keene, J. B., Kaltenback, A. J., Krumsiek, K.A.O., Morton, A. C., Murray, J. W., and Westberg-Smith, J., 1984. Oxygen isotope calibration of the onset of ice-rafting and history of glaciation in the North Atlantic region. Nature, 307:620-623.

Shackleton, N. J., and Kennett, J. P., 1975. Paleotemperature history of the Cenozoic and the initiation of Antarctic glaciation: oxygen and carbon isotope analyses in DSDP Sites 277,279 , and 281. In Kennett, J. P., Houtz, R. E., et al., Init. Repts. DSDP, 29: Washington (U.S. Govt. Printing Office), 743-755.

Shipboard Scientific Party, 1988. Site 704. In Ciesielski, P. F., Kristoffersen, Y., et al., Proc. ODP, Init. Repts., 114: College Station, TX (Ocean Drilling Program), 621-795.

Srinivasan, M. S., and Kennett, J. P., 1974. Secondary calcification of the planktonic foraminifer Neogloboquadrina pachyderma as a climatic index. Science, 186:630-632.

Williams, D. F., Sommer, M. A., II, and Bender, M. L., 1977. Carbon isotopic compositions of Recent planktonic foraminifers of the Indian Ocean. Earth Planet. Sci. Lett., 36:391-403.

Date of initial receipt: 12 April 1989

Date of acceptance: 17 November 1989

Ms 114B-152 


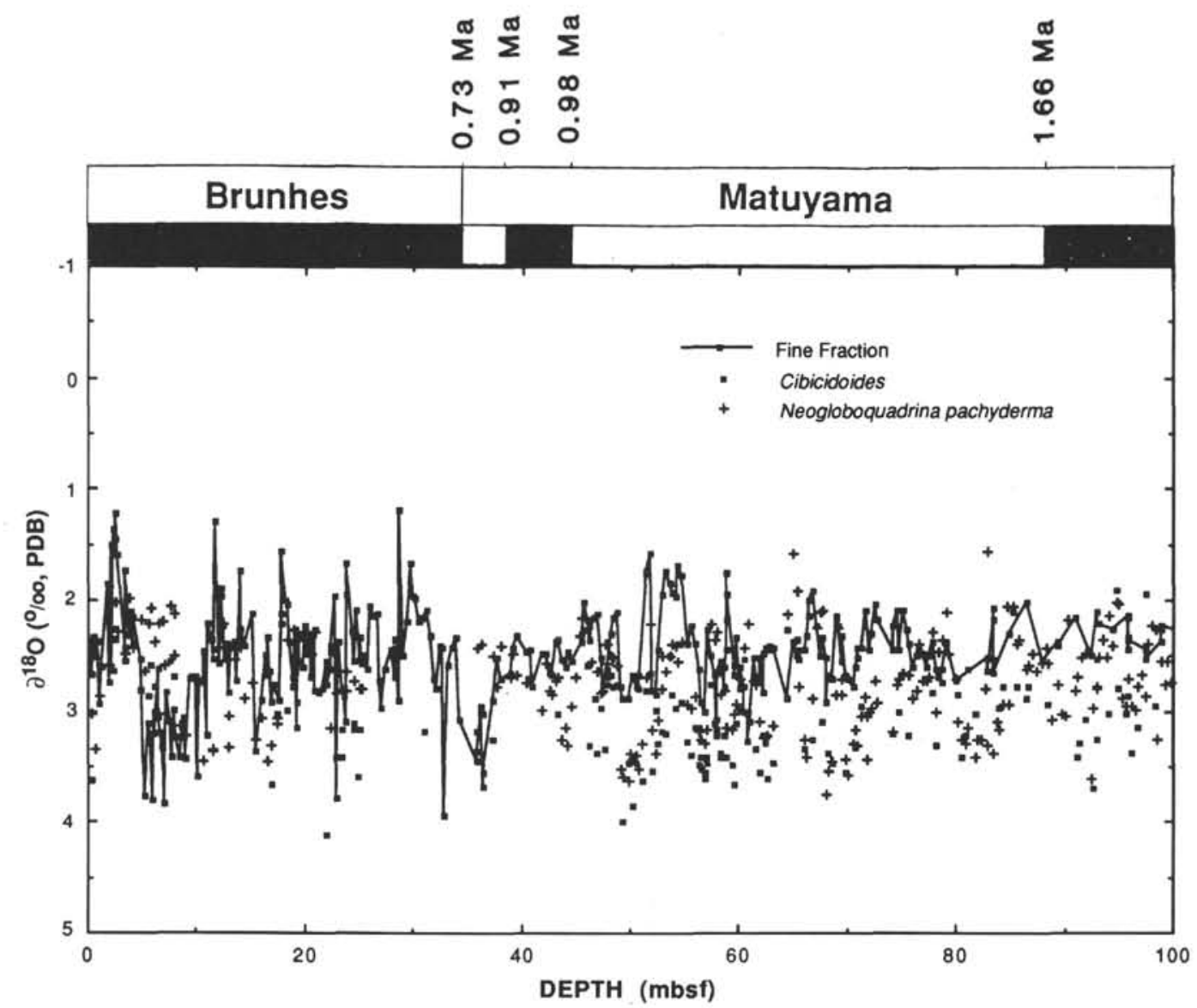

Figure 5. Comparison of $\delta^{18} \mathrm{O}$ records from the fine-fraction and benthic and planktonic foraminifers vs. depth, 0-300 mbsf. 


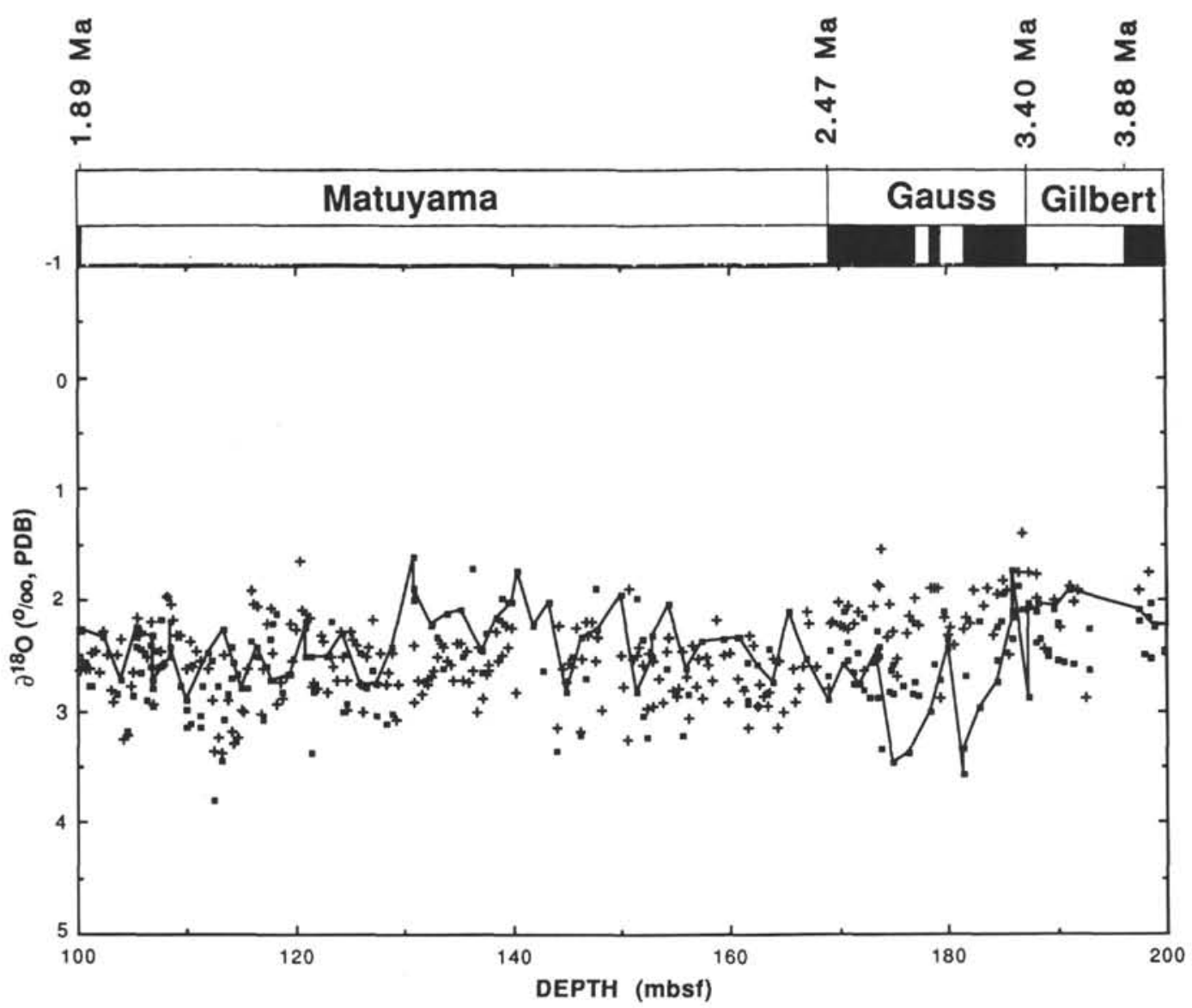

Figure 5 (continued). 


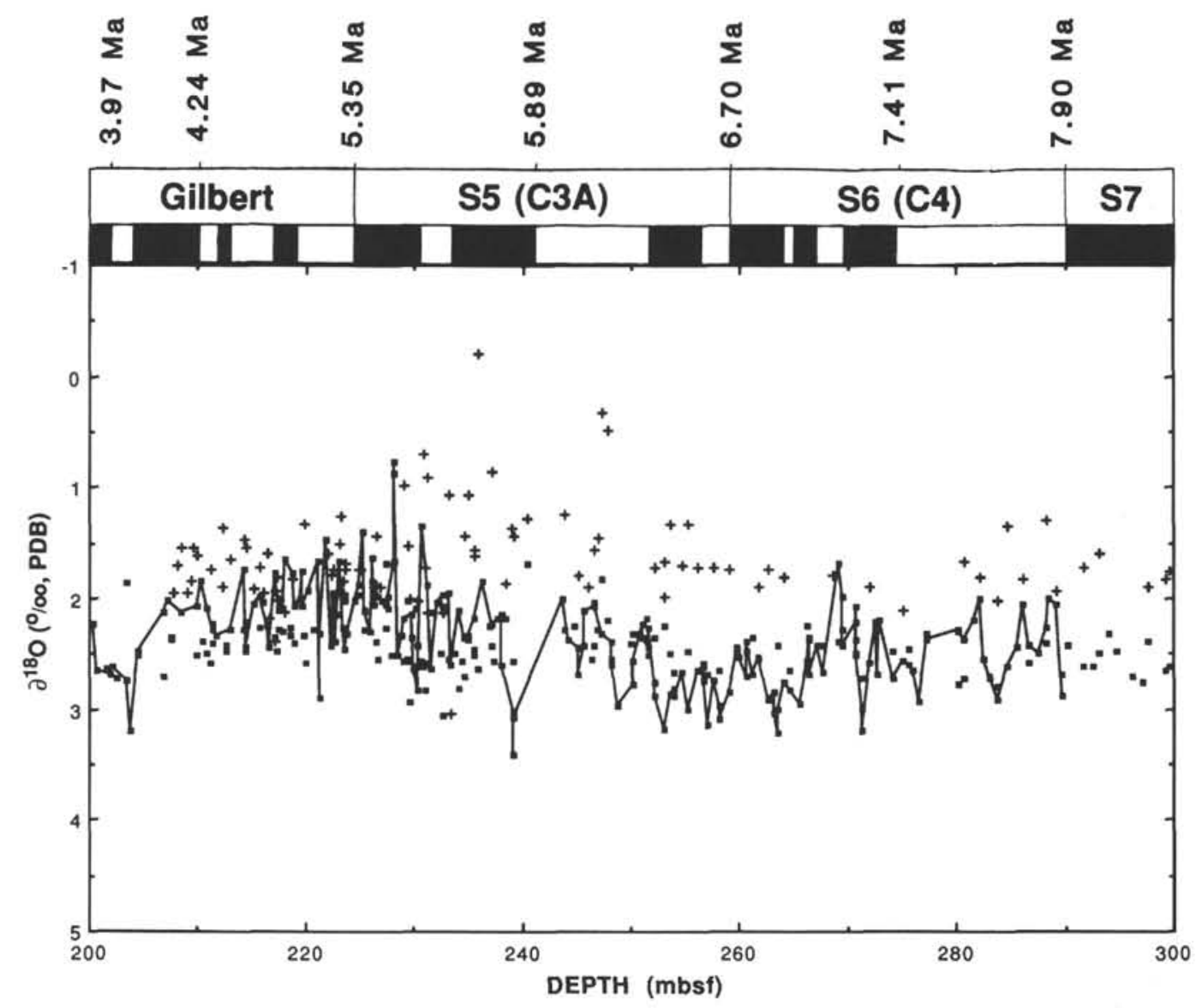

Figure 5 (continued). 


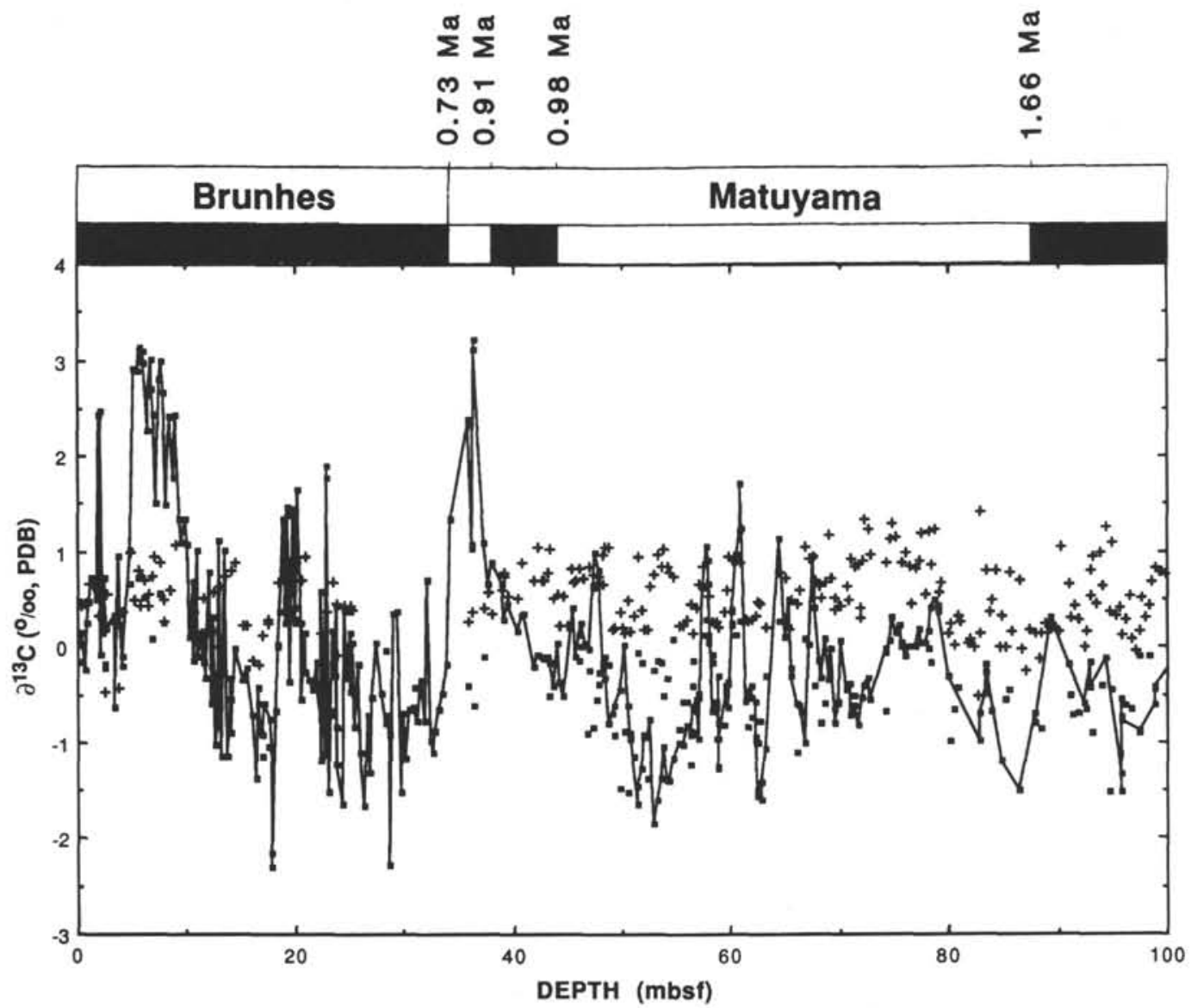

Figure 6. Comparison of $\delta^{13} \mathrm{C}$ records from the fine-fraction and benthic and planktonic foraminifers vs. depth, 0-300 mbsf. Open boxes = fine-fraction analyses; crosses = Neogloboquadrina pachyderma analyses; solid boxes $=$ Cibicidoides analyses. 


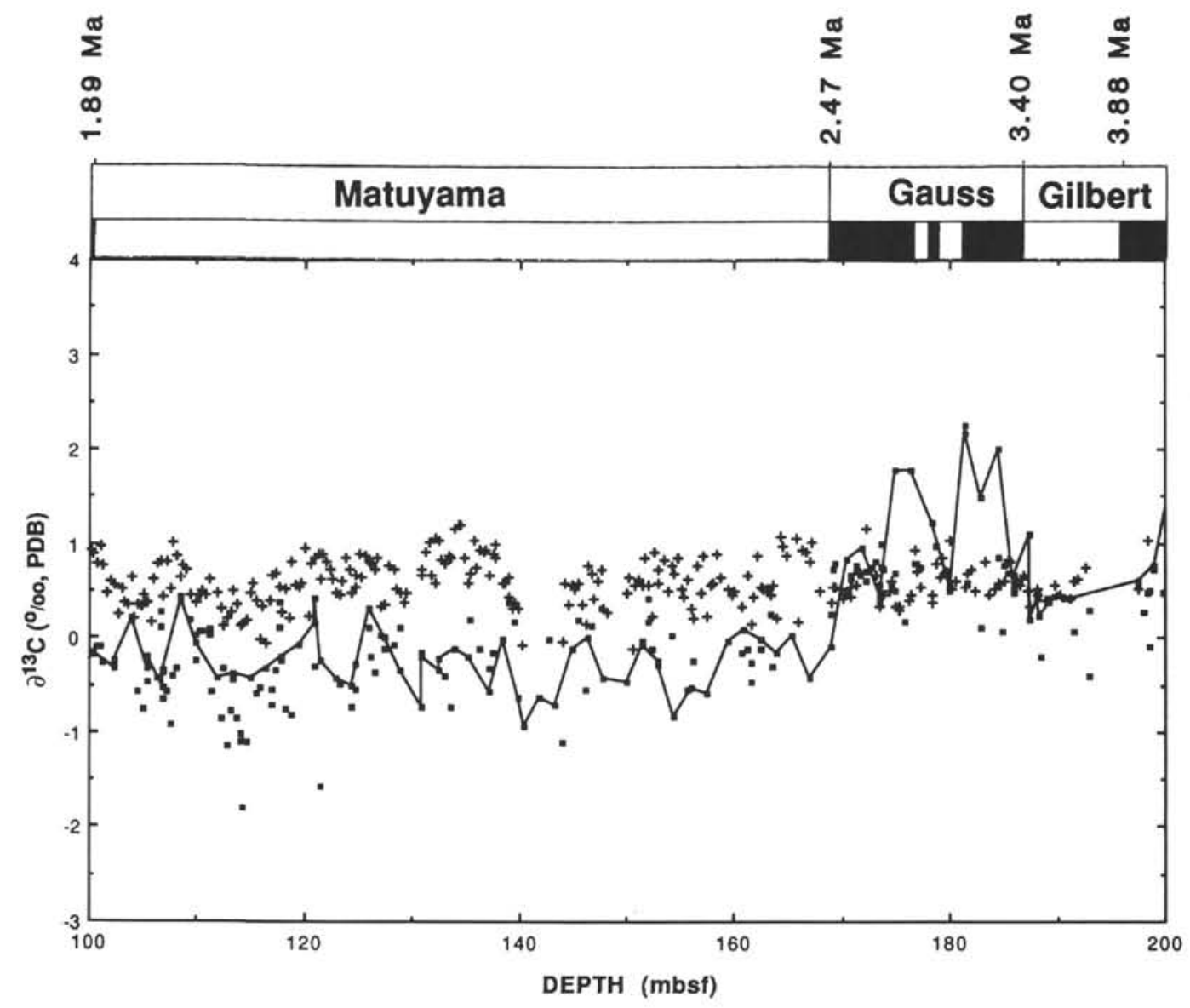

Figure 6 (continued). 


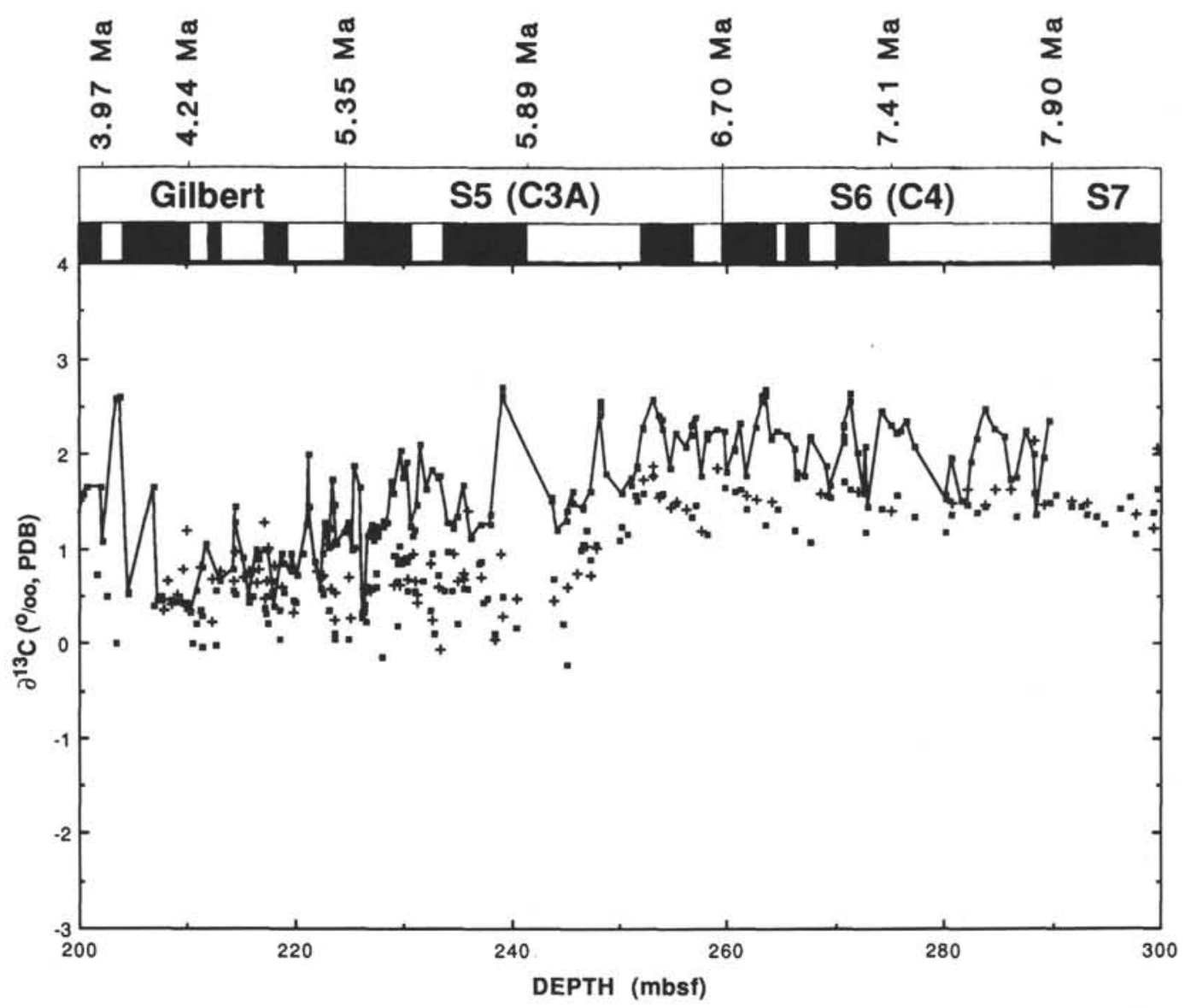

Figure 6 (continued). 
G. A. MEAD ET AL.

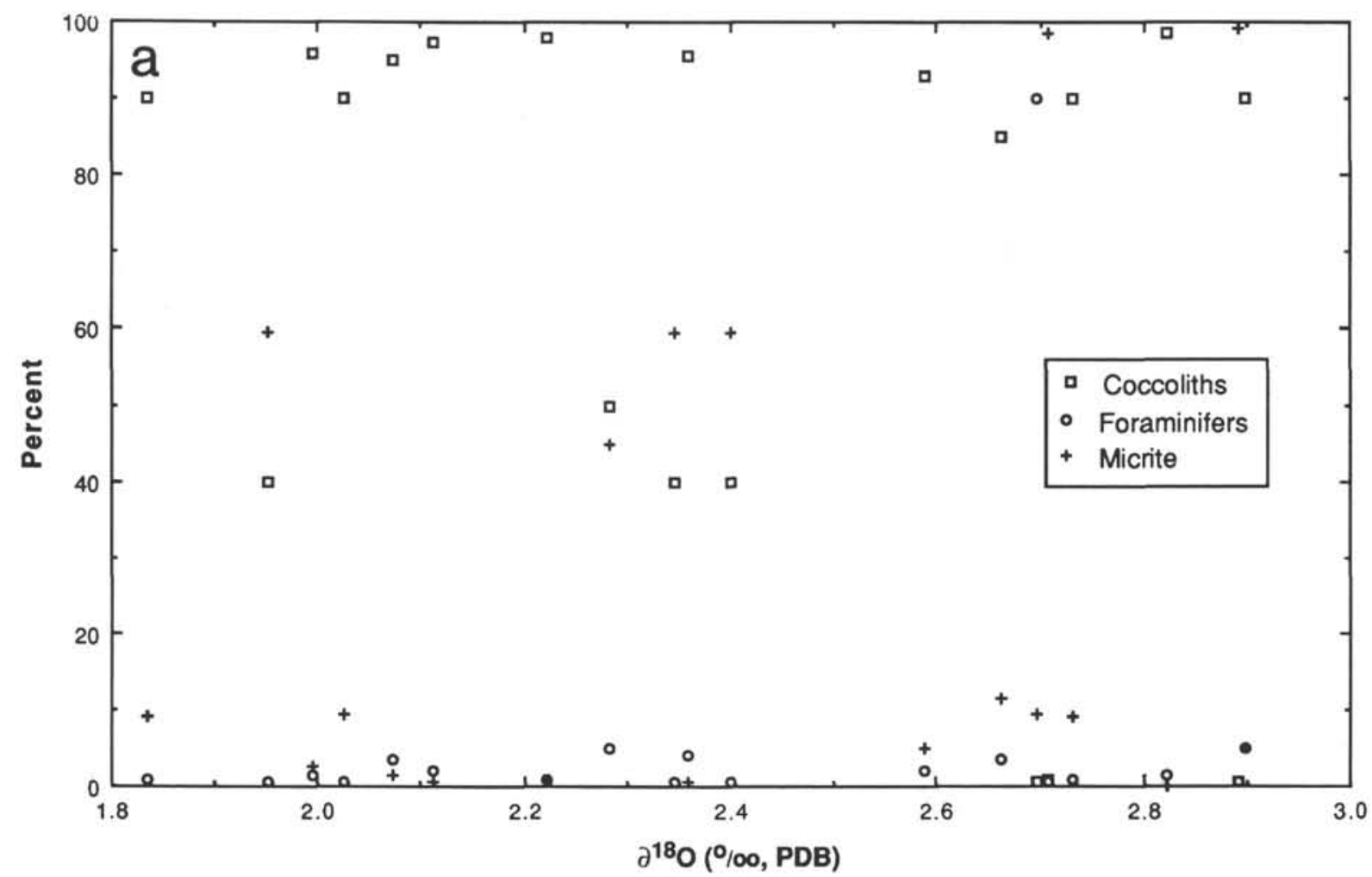

Figure 7. Fine-fraction composition vs. isotopes, Site 704. No correlation is visible between bulk fine-fraction composition and isotopic composition. A. $\delta^{18} \mathrm{O}$. B. $\delta^{13} \mathrm{C}$.

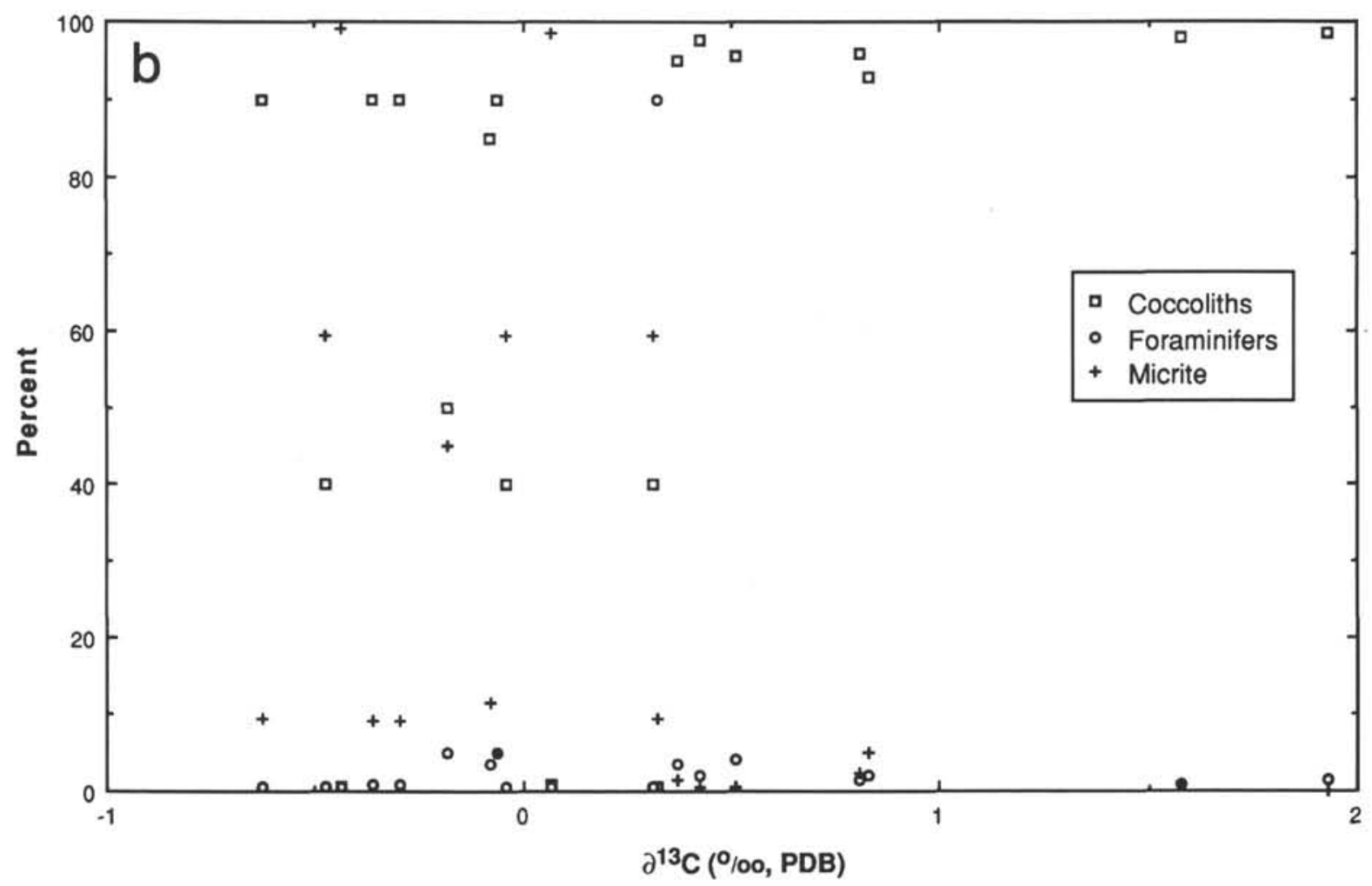

Figure 7 (continued). 